\title{
Two-step electroweak symmetry-breaking: theory meets experiment
}

\author{
Nicole F. Bell, ${ }^{a}$ Matthew J. Dolan, ${ }^{a}$ Leon S. Friedrich, ${ }^{a}$ Michael J. Ramsey-Musolf ${ }^{b, c, d}$ \\ and Raymond R. Volkas ${ }^{a}$ \\ ${ }^{a}$ ARC Centre of Excellence for Particle Physics at the Terascale, \\ School of Physics, The University of Melbourne, \\ Melbourne, VIC 3010, Australia \\ ${ }^{b}$ Amherst Center for Fundamental Interactions, \\ Department of Physics, University of Massachusetts Amherst, \\ Amherst, MA 01003, U.S.A. \\ ${ }^{c}$ Tsung-Dao Lee Institute and School of Physics and Astronomy, Shanghai Jiao Tong University, \\ 800 Dongchuan Road, Shanghai 200240, China \\ ${ }^{d}$ Kellogg Radiation Laboratory, California Institute of Technology, \\ Pasadena, CA 91125, U.S.A. \\ E-mail: n.bell@unimelb.edu.au, matthew.dolan@unimelb.edu.au, \\ leon.friedrich@unimelb.edu.au, mjrm@physics.umass.edu, \\ raymondv@unimelb.edu.au
}

ABSTRACT: We study the phenomenology of a hypercharge-zero SU(2) triplet scalar whose existence is motivated by two-step electroweak symmetry-breaking. We consider both the possibility that the triplets are stable and contribute to the dark matter density, or that they decay via mixing with the standard model Higgs boson. The former is constrained by disappearing charged track searches at the LHC and by dark matter direct detection experiments, while the latter is constrained by existing multilepton collider searches. We find that a two-step electroweak phase transition involving a stable triplet with a negative quadratic term is ruled out by direct detection searches, while an unstable triplet with a mass less than $230 \mathrm{GeV}$ is excluded at $95 \%$ confidence level.

Keywords: Beyond Standard Model, Higgs Physics

ARXiv EPrint: 2001.05335 


\section{Contents}

1 Introduction 1

2 Model 3

$2.1 \mathbb{Z}_{2}$ symmetric model 4

$2.2 \quad \mathbb{Z}_{2}$ broken model $\quad 5$

$\begin{array}{lll}2.3 & \text { Perturbative unitarity and perturbativity } & 6\end{array}$

$\begin{array}{lll}2.4 & \text { RGEs and running constraints } & 7\end{array}$

2.5 Phase transition requirements 8

2.6 Higgs diphoton rate 9

3 Stable triplet phenomenology 11

$\begin{array}{lll}3.1 & \text { Disappearing tracks } & 11\end{array}$

3.2 Dark matter direct detection 13

4 Unstable triplet phenomenology 16

$\begin{array}{lll}4.1 & \text { Production processes } & 16\end{array}$

$\begin{array}{lll}4.2 & \text { Decay channels } & 18\end{array}$

$\begin{array}{lll}4.3 & \text { Collider searches } & 20\end{array}$

$\begin{array}{ll}4.4 \text { Collider constraints } & 20\end{array}$

5 Conclusion 23

\section{Introduction}

The origin of the baryon asymmetry of the universe is a major open problem in particle physics and cosmology. Successful baryogenesis mechanisms require extensions to the standard model (SM), as it has neither enough charge-parity $(\mathrm{CP})$ violation nor does it provide the necessary out-of-equilibrium conditions. Electroweak baryogenesis provides one possible solution, and is particularly attractive as its association with new electroweak scale physics means it is testable experimentally via collider searches [1] and electric dipole moment (EDM) measurements (for a review, see, e.g. [2]).

There has been recent interest in the possibility of multi-step electroweak phase transitions [3-8]. In such scenarios the electroweak phase transition consists of multiple transitions, where initially an exotic scalar charged under $\mathrm{SU}(2)$ gains a vacuum expectation value (VEV) before a second transition to the SM Higgs phase takes place. This scenario is attractive because the extended scalar sector has enough freedom to support a strongly first order transition, and the new CP violating interactions can be partially hidden in the new scalar sector in order to avoid tight EDM constraints. Two step phase transitions have 
been examined for a range of extended scalar sectors, including $\mathrm{SU}(2)$ triplet scalar extensions [3, 8], two Higgs doublet models [5] and coloured scalar extensions [4, 7]. Two-step transitions have also been studied in the context of scalar sector extensions containing real or complex singlets [9-15]. In these scenarios, electroweak symmetry breaking occurs only once - during the final transition to the present Higgs phase.

The simplest ${ }^{1}$ model that can feature the desired two step electroweak symmetry breaking transition is the real $\mathrm{SU}(2)$ triplet scalar $\Sigma \sim(1,3,0)$ extension to the $\mathrm{SM}$ (the $\Sigma \mathrm{SM}$ ). Such an electroweak scale triplet may arise from the breaking of a high-scale GUT, e.g., the $\mathbf{2 1 0}$ of $\mathrm{SO}(10)$ [16]. The phase transition structure of the $\Sigma \mathrm{SM}$ has been examined by refs. $[3,8,17]$. While $[3,17]$ focused on phase transitions rather than collider physics, [8] has studied the impact on collider phenomenology in more detail. However, they consider a dimension-5 effective operator involving the triplet that significantly modifies the phenomenology relative to the minimal triplet model that we study. The general phenomenology of minimal hypercharge-zero $\mathrm{SU}(2)$ triplet scalar extensions has been studied extensively [18-21], with a significant focus on the prospects of having the neutral component of the triplet be stable and thus provide some or all of the dark matter (DM) density [22-28].

Ref. [18] examines the prospect of constraining triplet scalars via measurements of the Higgs diphoton decay rate, disappearing track searches, and collider production searches. However, as ref. [18] was published prior to first collisions at the LHC, no lower bounds on the triplet mass were set beyond those following from searches at the LEP collider. The more recent studies [20, 21] consider corrections to SM Higgs production rates and decay processes, and do not obtain a lower bound on the triplet arising from the production and decay of the triplets at the LHC. In the scenario where the neutral component of the triplet is stable, existing DM direct detection constraints severely restrict the size of the triplet's coupling to the SM Higgs. However, in order for the neutral component of the triplet make up a significant fraction of the DM density it is required to have a mass $\sim 2 \mathrm{TeV}$. In contrast, acquiring a multi-step electroweak phase transition requires the mass to be electroweak-scale $\lesssim 1 \mathrm{TeV}$. Thus the parameter-space relevant to multi-step phase transitions will only ever result in the triplet contributing a small fraction of the DM density and is generally not thoroughly explored in triplet scalar DM studies.

We extend the previous examinations of $\mathrm{SU}(2)$ triplet scalar phenomenology in a number of ways. Firstly, we show that if the neutral triplet is stable or very long lived, then existing disappearing track searches constrain the mass of the triplet to be larger than $\sim 250 \mathrm{GeV}$. Secondly, we examine the scenario where the neutral component of the triplet is both stable and hypothetically constitutes a portion of the dark matter. We show that the parameter-space favourable for a multi-step electroweak phase transition is ruled out by dark matter direct detection experiments. Finally, we demonstrate that if the triplet is unstable, existing LHC multilepton searches place a lower bound on its mass of around $230 \mathrm{GeV}$. Utilising multilepton searches to constrain triplets has previously been examined by refs. [29] and [30-32] in the context of a triplet extended super-symmetric standard model, and a type-II seesaw model, respectively.

\footnotetext{
${ }^{1}$ Simplest in the sense that it has the fewest additional physical particles, and the fewest new parameters present without imposing additional symmetries.
} 
The above dark matter direct detection constraint implies that the neutral member of the triplet must be allowed to decay if it is to be relevant for 2-step EWSB. The stability of the neutral triplet in the $\Sigma \mathrm{SM}$ arises from the imposition of a $\Sigma \rightarrow-\Sigma$ discrete $\mathbb{Z}_{2}$ symmetry on the model. This symmetry can be broken explicitly by a term in the Lagrangian, so that the neutral triplet can decay and the dark matter constraints are avoided. However, the collider production constraints remain relevant. The advent of additional LHC data will increase the reach in both mass and coupling, thereby providing a powerful probe of this scenario.

\section{Model}

We extend the Standard Model by adding a real scalar field $\Sigma$ transforming as $(1,3,0)$ under the $\mathrm{SU}(3) \times \mathrm{SU}(2) \times \mathrm{U}(1)_{Y} \mathrm{SM}$ gauge group. We consider the most general renormalisable scalar potential,

$$
\begin{aligned}
V_{0}(H, \Sigma)= & -\mu_{H}^{2} H^{\dagger} H-\frac{1}{2} \mu_{\Sigma}^{2} \operatorname{Tr}\left(\Sigma^{2}\right)+\lambda_{H}\left(H^{\dagger} H\right)^{2}+\frac{1}{4} b_{4}\left[\operatorname{Tr}\left(\Sigma^{2}\right)\right]^{2} \\
& +\frac{1}{\sqrt{2}} a_{1} H^{\dagger} \Sigma H+\frac{1}{2} a_{2} \operatorname{Tr}\left(\Sigma^{2}\right) H^{\dagger} H
\end{aligned}
$$

where $H$ is the SM scalar Higgs doublet, and we use the notation

$$
\Sigma=\left[\begin{array}{cc}
\frac{1}{\sqrt{2}}\left(\Sigma^{0}+v_{\Sigma}\right) & \Sigma^{+} \\
\Sigma^{-} & -\frac{1}{\sqrt{2}}\left(\Sigma^{0}+v_{\Sigma}\right)
\end{array}\right], \quad H=\left[\begin{array}{c}
H^{+} \\
\frac{1}{\sqrt{2}}\left(v_{H}+H^{0}+i A^{0}\right)
\end{array}\right] .
$$

For real triplets, terms in the potential proportional to $\operatorname{Tr}\left(\Sigma^{4}\right)$ and $H^{\dagger} \Sigma^{2} H$ can be absorbed into the $\left[\operatorname{Tr}\left(\Sigma^{2}\right)\right]^{2}$ and $\operatorname{Tr}\left(\Sigma^{2}\right) H^{\dagger} H$ terms and simply redefine $b_{4}$ and $a_{2}$. We only consider negative quadratic coefficients for the triplet and the Higgs doublet. To ensure that the potential is bounded from below we require

$$
\lambda_{H}>0, b_{4}>0, a_{2} \geq-2 \sqrt{\lambda_{H} b_{4}} .
$$

Additionally, the vacuum at zero temperature must approximate the SM Higgs-phase within errors, so that

$$
m_{H} \simeq 125 \mathrm{GeV}, v_{H} \simeq 246 \mathrm{GeV} .
$$

The VEV of the triplet $v_{\Sigma}$ is constrained by precision electroweak measurements as it contributes to the $\rho$ parameter. At tree level the correction to the $\rho$ parameter is

$$
\delta \rho=\rho-1=\frac{4 v_{\Sigma}^{2}}{v_{H}^{2}} .
$$

The current measurement of $\rho=1.00039 \pm 0.00019$ [33] requires $v_{\Sigma} \lesssim 3 \mathrm{GeV}$.

We consider two scenarios: a model where we impose a $\Sigma \rightarrow-\Sigma$ discrete $\mathbb{Z}_{2}$ symmetry on the theory, which eliminates the $a_{1}$ coupling, and a model with no such symmetry, where $a_{1} \neq 0$. In the remainder of this section we discuss the notation and selection of parameters in each scenario before moving on to discuss perturbativity constraints, electroweak phase transition requirements, and corrections to the SM Higgs diphoton rate. 


\section{$2.1 \quad \mathbb{Z}_{2}$ symmetric model}

With the $\Sigma \rightarrow-\Sigma$ symmetry imposed on the theory, the potential has four permissible types of extrema [18]:

1. $v_{H}^{2}=0, v_{\Sigma}^{2}=0$

2. $v_{H}^{2}=0, v_{\Sigma}^{2}=\frac{\mu_{\Sigma}^{2}}{b_{4}}$

3. $v_{H}^{2}=\frac{\mu_{H}^{2}}{\lambda_{H}}, v_{\Sigma}^{2}=0$

4. $v_{H}^{2}=\frac{4 b_{4} \mu_{H}^{2}-2 a_{2} \mu_{\Sigma}^{2}}{4 \lambda_{H} b_{4}-a_{2}{ }^{2}}, v_{\Sigma}^{2}=\frac{4 \lambda_{H} \mu_{\Sigma}^{2}-2 a_{2} \mu_{H}^{2}}{4 \lambda_{H} b_{4}-a_{2}{ }^{2}}$

Only the latter two can yield SM-like minima, since $v_{H} \neq 0$. However, the fourth possibility results in a physical charged scalar that is massless at tree-level. This is due to the fact that the $\mathbb{Z}_{2}$ symmetric potential features only $\operatorname{Tr}\left(\Sigma^{2}\right)$ terms, leading to an accidental $\mathrm{SO}(3)$ global symmetry which rotates the components of $\Sigma$ amongst themselves but under which $H$ is a singlet. This symmetry is spontaneously broken when the triplet gains a VEV, yielding a charged pseudo-Goldstone scalar boson.

Therefore we focus on the scenario where the zero temperature potential has a global minimum of the third type. This extremum is a local minimum when the parameters satisfy

$$
\frac{\mu_{H}^{2}}{\lambda_{H}}=v_{H}^{2}>2 \frac{\mu_{\Sigma}^{2}}{a_{2}}
$$

and is the global minimum when

$$
\frac{\mu_{H}^{4}}{\lambda_{H}}>\frac{\mu_{\Sigma}^{4}}{b_{4}}
$$

The Higgs couplings then take their SM values, $\lambda_{H}=\frac{m_{H}^{2}}{2 v_{H}^{2}}$ and $\mu_{H}^{2}=\frac{m_{H}^{2}}{2}$. The potential has three free parameters $a_{2}, \mu_{\Sigma}^{2}$, and $b_{4}$. We swap $a_{2}$ for the triplet mass using the relation

$$
m_{\Sigma^{0}}^{2}=-\mu_{\Sigma}^{2}+\frac{1}{2} a_{2} v_{H}^{2}
$$

The form of the $\mathbb{Z}_{2}$-symmetric potential has the triplet components being degenerate at tree-level. However, radiative corrections lead to a small mass splitting between the neutral and charged components [22],

$$
\Delta m_{\Sigma}=m_{\Sigma^{+}}-m_{\Sigma^{0}}=\frac{\alpha_{2} m_{\Sigma^{0}}}{4 \pi}\left[f\left(\frac{m_{W}}{m_{\Sigma^{0}}}\right)-c_{W}^{2} f\left(\frac{m_{Z}}{m_{\Sigma^{0}}}\right)\right]>0,
$$

where $c_{W}$ is the cosine of the weak mixing angle and,

$$
f(r)=-\frac{r}{4}\left[2 r^{3} \ln r+\left(r^{2}-4\right)^{3 / 2} \ln \left(\frac{r^{2}-2-r \sqrt{r^{2}-4}}{2}\right)\right] .
$$

The mass splitting decreases with increasing triplet mass, and in the limit $\frac{m_{\Sigma^{0}}}{m_{Z}} \gg 1$ the mass splitting approaches $\Delta m_{\Sigma}=166 \mathrm{MeV}$. While the neutral component remains stable, 
this small splitting allows the charged component to decay via an off shell $W^{ \pm}$into the neutral component and either a low energy pion or a light charged lepton and neutrino. The widths of the associated decays are given by $[22,34]$

$$
\begin{array}{ll}
\Sigma^{+} \rightarrow \Sigma^{0} \pi^{+}: & \Gamma_{\pi}=\frac{2 G_{\mathrm{F}}^{2}\left|V_{u d}\right|^{2} \Delta m_{\Sigma}^{3} f_{\pi}^{2}}{\pi} \sqrt{1-\frac{m_{\pi}^{2}}{\Delta m_{\Sigma}^{2}}} \\
\Sigma^{+} \rightarrow \Sigma^{0} e^{+} \nu_{e}: & \Gamma_{e}=\frac{2 G_{\mathrm{F}}^{2} \Delta m_{\Sigma}^{5}}{15 \pi^{3}} \\
\Sigma^{+} \rightarrow \Sigma^{0} \mu^{+} \nu_{\mu}: & \Gamma_{\mu}=K\left(\frac{m_{\mu}}{\Delta m_{\Sigma}}\right) \Gamma_{e},
\end{array}
$$

where $f_{\pi} \simeq 131 \mathrm{MeV}$ and

$$
K(x)=\frac{15}{2} x^{4} \log \frac{1+\sqrt{1-x^{2}}}{x}-\frac{1}{2} \sqrt{1-x^{2}}\left(8 x^{4}+9 x^{2}-2\right) .
$$

\section{$2.2 \quad \mathbb{Z}_{2}$ broken model}

Turning on the $\mathbb{Z}_{2}$-breaking $a_{1}$ term changes the results of the previous subsection. In particular, for the third type of extremum the triplet gains a small induced VEV from the $H^{2} \Sigma$ term, with the potential now minimised by

$$
\begin{aligned}
v_{\Sigma} & =\frac{a_{1} v_{H}^{2}}{-4 \mu_{\Sigma}^{2}+2 v_{H}^{2} a_{2}+4 v_{\Sigma}^{2} b_{4}} \\
& \simeq \frac{a_{1} v_{H}^{2}}{-4 \mu_{\Sigma}^{2}+2 v_{H}^{2} a_{2}}=\frac{a_{1} v_{H}^{2}}{4 m_{\Sigma^{0}}^{2}} \\
v_{H}^{2} & =\frac{\mu_{H}^{2}}{\lambda_{H}}+\frac{a_{1} v_{\Sigma}-a_{2} v_{\Sigma}^{2}}{2 \lambda_{H}} \simeq \frac{\mu_{H}^{2}}{\lambda_{H}},
\end{aligned}
$$

where $m_{\Sigma^{0}}$ is the mass of the triplet in the $\mathbb{Z}_{2}$ symmetric case, eq. (2.8), and the approximations hold when the triplet VEV is small.

Additionally the $a_{1}$ term in the potential and the triplet's non-zero VEV result in new mass terms leading to mixing between the neutral component of the triplet and SM Higgs,

$$
\begin{aligned}
\mathcal{L} & \supset \frac{1}{2}\left(\begin{array}{ll}
H^{0} & \Sigma^{0}
\end{array}\right) \mathcal{M}_{N}\left(\begin{array}{c}
H^{0} \\
\Sigma^{0}
\end{array}\right), \\
& =\frac{1}{2}\left(\begin{array}{ll}
h_{1} & h_{2}
\end{array}\right)\left(\begin{array}{cc}
m_{h_{1}}^{2} & 0 \\
0 & m_{h_{2}}^{2}
\end{array}\right)\left(\begin{array}{l}
h_{1} \\
h_{2}
\end{array}\right),
\end{aligned}
$$

where we have introduced the neutral scalar mass matrix,

$$
\mathcal{M}_{N}=\left(\begin{array}{cc}
2 \lambda_{H} v_{H}^{2} & a_{2} v_{H} v_{\Sigma}-\frac{1}{2} a_{1} v_{H} \\
a_{2} v_{H} v_{\Sigma}-\frac{1}{2} a_{1} v_{H} & -\mu_{\Sigma}^{2}+\frac{1}{2} a_{2} v_{H}^{2}+3 b_{4} v_{\Sigma}^{2}
\end{array}\right)
$$

and the mass basis,

$$
\left(\begin{array}{l}
h_{1} \\
h_{2}
\end{array}\right)=\left(\begin{array}{cc}
\cos \theta_{N} & -\sin \theta_{N} \\
\sin \theta_{N} & \cos \theta_{N}
\end{array}\right)\left(\begin{array}{c}
H^{0} \\
\Sigma^{0}
\end{array}\right) .
$$


The neutral scalar mixing angle $\theta_{N}$ is defined such that $h_{1}$ is the particle that consists primarily of $H^{0}$. As we require $v_{\Sigma} \lesssim 3 \mathrm{GeV}$, and as the off-diagonal term is directly proportional to $v_{\Sigma}$, the mixing term is necessarily small. Hence, unless the scalars are nearly degenerate the mixing angle will also be small. It is then sufficient to use the SM values for $\mu_{H}$ and $\lambda_{H}$ in order to produce a SM-like Higgs with $m_{h_{1}} \approx 125 \mathrm{GeV}$ and $v_{H} \approx 246 \mathrm{GeV}$. The potential then has four free parameters: $\mu_{\Sigma}^{2}, a_{2}, a_{1}$, and $b_{4}$. We will fix $a_{2}$ and $a_{1}$ by requiring that we get values for $m_{h_{2}}$ and $v_{\Sigma}$, as given by diagonalising $\mathcal{M}_{N}$ and solving eq. (2.13), respectively.

There will also be mixing in the charged scalar sector,

$$
\mathcal{L} \supset\left(H^{-} \Sigma^{-}\right) \mathcal{M}_{C}\left(\begin{array}{c}
H^{+} \\
\Sigma^{+}
\end{array}\right)=\left(G^{-} h^{-}\right)\left(\begin{array}{cc}
0 & 0 \\
0 & m_{h^{+}}^{2}
\end{array}\right)\left(\begin{array}{c}
G^{+} \\
h^{+}
\end{array}\right),
$$

where

$$
\begin{aligned}
\mathcal{M}_{C} & =\frac{a_{1}}{4}\left(\begin{array}{cc}
4 v_{\Sigma} & 2 v_{H} \\
2 v_{H} & v_{H}^{2} / v_{\Sigma}
\end{array}\right) \\
\left(\begin{array}{c}
G^{+} \\
h^{+}
\end{array}\right) & =\left(\begin{array}{cc}
\cos \theta_{C} & -\sin \theta_{C} \\
\sin \theta_{C} & \cos \theta_{C}
\end{array}\right)\left(\begin{array}{c}
H^{+} \\
\Sigma^{+}
\end{array}\right), \quad \sin \theta_{C}=\frac{v_{\Sigma}}{\sqrt{v_{\Sigma}^{2}+\frac{1}{4} v_{H}^{2}}} .
\end{aligned}
$$

The field $G^{+}$is the massless charged unphysical Goldstone boson and $h^{+}$is a physical charged scalar that consists primarily of the charged triplet component $\Sigma^{+}$.

In the limit $v_{\Sigma} \rightarrow 0$ we re-obtain the $\mathbb{Z}_{2}$ symmetric model and the masses of the scalars approach the values they would have had in the absence of mixing, $m_{h_{1}} \rightarrow m_{H}$, $m_{h_{2}} \rightarrow m_{\Sigma^{0}}$, and $m_{h^{+}} \rightarrow m_{\Sigma^{+}}$. For simplicity, we will use the notation of the $\mathbb{Z}_{2}$ broken model to identify particles and masses throughout the remainder of the paper, even if there is no mixing. Note that this limiting behaviour means that the radiative mass splitting discussed in the previous subsection will become important for very small $v_{\Sigma}$. However, unless $v_{\Sigma} \lesssim 10^{-3} \mathrm{GeV}[18]$ the charged scalar will primarily decay via its mixing with the charged Goldstone boson into pairs of fermions or $W^{ \pm} Z^{(*)}$, and not via the decays discussed in the previous section. Hence, unless $v_{\Sigma}$ is very small the decays will not be sensitive to the radiative mass splitting. We discuss the unstable triplet decays in detail in section 4.2.

\subsection{Perturbative unitarity and perturbativity}

Requiring that our couplings satisfy perturbative unitarity, i.e. that the tree-level high energy $2 \rightarrow 2$ scattering amplitudes remain unitary, leads to the constraints [20, 21],

$$
\begin{aligned}
\left|a_{2}\right| & \leq 8 \pi \\
\left|\lambda_{H}\right|,\left|b_{4}\right| & \leq 4 \pi \\
\left|6 \lambda_{H}+5 b_{4} \pm \sqrt{\left(6 \lambda_{H}-5 b_{4}\right)^{2}+12 a_{2}}\right| & \leq 16 \pi
\end{aligned}
$$

where we have utilised the unitarity constraint with $\left|\operatorname{Re}\left(a_{0}\right)\right| \leq \frac{1}{2}$. Combining these constraints with the requirement that the potential be bounded from below, eq. (2.3), the 
constraints on the couplings become,

$$
\begin{aligned}
0 & \leq \lambda_{H} \leq \frac{4}{3} \pi \\
0 & \leq b_{4} \leq \frac{8}{5} \pi \\
\left|a_{2}\right| & \leq \sqrt{10\left(\lambda_{H}-\frac{4}{3} \pi\right)\left(b_{4}-\frac{8}{5} \pi\right)} \lesssim 4.54 \pi .
\end{aligned}
$$

While well defined, the perturbative unitarity requirement is separate from the requirement that the scalar couplings be perturbative. The definition of perturbativity is somewhat subjective. One method of defining a perturbativity bound is via the renormalization group equations (RGEs). In the SM at one-loop level, the Higgs quartic coupling features a Laundau pole at high energy. On the other hand, the two-loop RGEs instead have the quartic coupling approaching a fixed point $\lambda_{H}(\mu) \rightarrow \lambda_{H}^{\mathrm{FP}} \approx 12[35,36]$. When $\lambda_{H}=\lambda_{H}^{\mathrm{FP}}$ the two-loop contributions to the RGEs cancel the one-loop terms, therefore the fixed point provides a value of the coupling at which perturbativity begins to break down. This same behaviour is present in the real triplet scalar extended standard model. Therefore, following refs. [35-38], we impose the requirement,

$$
\lambda<\frac{\lambda^{\mathrm{FP}}}{3}, \quad \lambda \in\left\{\lambda_{H}, b_{4}, a_{2}\right\}
$$

where $\lambda^{\mathrm{FP}}$ is the fixed point of each of the scalar couplings. We utilise the SARAH 4.14.3 [39] package, which has an implementation of the real triplet extension, to evaluate the two-loop RGEs. We find that for a wide range of initial conditions, the scalar couplings approach the fixed points,

$$
\lambda_{H}^{\mathrm{FP}} \approx 12, \quad b_{4}^{\mathrm{FP}} \approx 6, \quad a_{2}^{\mathrm{FP}} \approx 23 .
$$

Thus, our perturbativity requirement is,

$$
\lambda_{H}<4, \quad b_{4}<2, \quad a_{2}<7.7 .
$$

With the exception of the Higgs quartic coupling, this perturbativity condition is significantly more restrictive than the perturbative unitarity requirement from eq. (2.20).

\subsection{RGEs and running constraints}

We also require that the perturbativity and perturbative unitarity conditions continue to be satisfied at higher energy scales, up to some cutoff energy $\Lambda$. In particular, if a set of parameters lies near the non-perturbative region and one uses the RGEs to run the couplings they may rapidly become non-perturbative even at relatively low energies $(\sim 1 \mathrm{TeV})$. The choice of cutoff energy significantly impacts the amount of parameter-space available. Figure 1 shows how the available parameter-space depends on the energy cutoff. Requiring that the perturbativity conditions are satisfied up to $\Lambda=10^{6} \mathrm{GeV}$ or higher removes a large chunk of the available parameter-space. We consider the requirement that the couplings continue to be perturbative up to at least $\Lambda=m_{h_{2}}, m_{h^{+}} \sim 1 \mathrm{TeV}$ to be the 

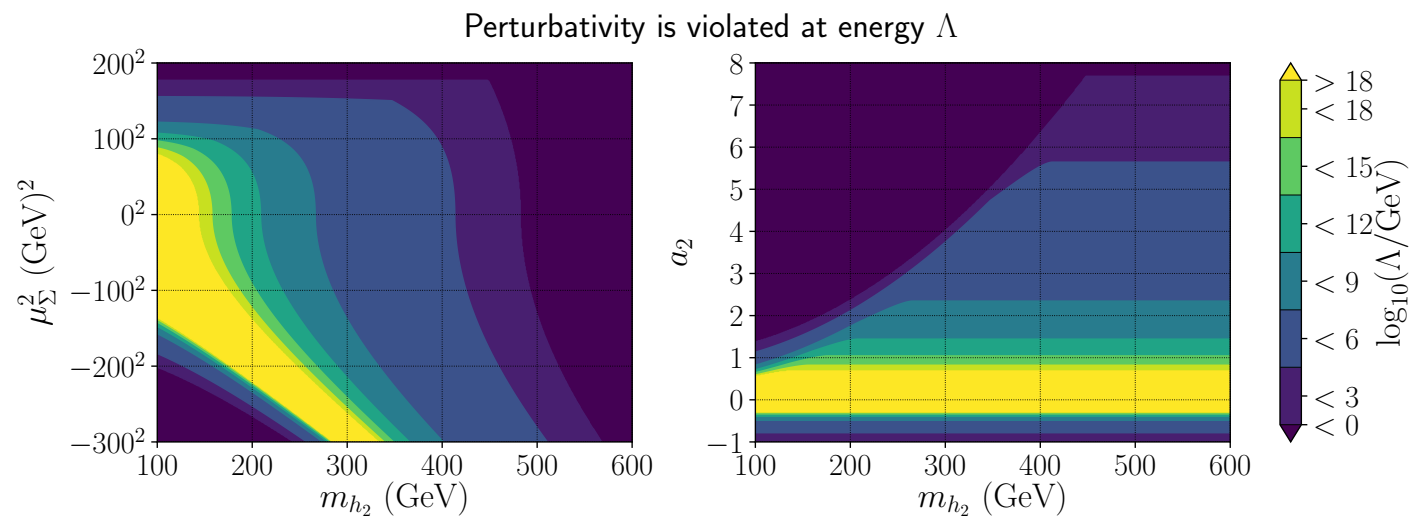

Figure 1. Contour plots showing the energies at which the perturbativity and perturbative unitarity constraints are violated as a function of $m_{h_{2}}$ and either $\mu_{\Sigma}^{2}$ (left) or $a_{2}$ (right). The darkest shade corresponds to the region of parameter-space where the conditions are not satisfied by the initial choice of parameters, with no running necessary. Conversely, the lightest shade corresponds to the region where the conditions are still satisfied after running the couplings up to very high energies. For each point we have set $v_{\Sigma}=0$, and $b_{4}$ to the minimal value allowed by eq. (2.7). This choice of $b_{4}$ was found to maximise the energy at which the conditions were first violated.

bare-minimum requirement that we will impose for the remainder of the paper, though we will also consider more restrictive higher energy cut-offs. However, if there are additional light particles $\left(m<10^{6} \mathrm{GeV}\right)$ that strongly couple to the SM Higgs or triplet, then they may significantly modify the running. Hence, even requiring perturbativity and perturbative unitarity only up to $\Lambda=10^{6} \mathrm{GeV}$ may be excessive if one expects such new physics.

\subsection{Phase transition requirements}

We study a model where, in the early universe, electroweak symmetry breaking occurs via a transition from the electroweak symmetric minimum to a minimum where the scalar triplet gains a VEV. A subsequent transition then takes us to the regular SM-like Higgs phase at a lower temperature. Requiring such a multi-step electroweak phase transition leads to constraints on the scalar potential parameters.

An important necessary condition is that the triplet should have a negative quadratic coefficient: $-\mu_{\Sigma}^{2}<0$. To see this, consider the opposite situation, that $-\mu_{\Sigma}^{2}>0$, where we deal with the $\mathbb{Z}_{2}$-symmetric model first. At finite temperature, the tendency is for a quadratic coefficient to gain a positive contribution so that $-\mu_{\Sigma}^{2}=\left|\mu_{\Sigma}^{2}\right| \rightarrow\left|\mu_{\Sigma}^{2}\right|+a T^{2}$, where $a>0 .^{2}$ In isolation, this effect goes against our desire for $\Sigma$ to have a nonzero VEV at finite temperature and thus participate in a two-step electroweak phase transition. The only way out is for a sufficiently large negative quadratic coefficient to be induced from a negative $a_{2}$ coupling such that the effective quadratic coefficient is negative: $\left|\mu_{\Sigma}^{2}\right|+a T^{2}+\frac{1}{2} a_{2} v_{H}^{2}<0$. But if this were the case, then at zero temperature the large and negative $a_{2}$ would induce a

\footnotetext{
${ }^{2}$ If there is a large negative $a_{2}$ coupling it is possible for the thermal term to be negative, leading to symmetry non-restoration. However, in our model this is incompatible with the requirement that the potential be bounded from below, eq. (2.3).
} 
large triplet VEV, which is ruled out from the $\rho$-parameter bound. Thus the opposite choice of $-\mu_{\Sigma}^{2}<0$ is the only viable possibility, and we adopt it as a necessary though not sufficient condition to have an acceptable two-step electroweak phase transition. ${ }^{3}$ This is consistent with the parameter space explored in previous multi-step phase transition models [4-7], particularly refs. $[3,8,17]$. The $\mathbb{Z}_{2}$-broken case follows similarly, with the only change being that the triplet gains a small induced VEV at zero temperature from the cubic $a_{1}$ term. Requiring that the VEV be small necessitates that $a_{1}$ is small, such that it has no significant impact on early universe phase transitions aside from breaking the $\mathbb{Z}_{2}$ symmetry.

A rigorous treatment of the finite temperature effective potential and early universe phase transitions is non-trivial, with significant theoretical and technical issues remaining to be addressed. In particular the typical phase transition treatments are gauge dependent [40]. However, even one-loop gauge-independent treatments lead to results that differ from current lattice simulations [40, 41]. Accordingly, it is difficult to make precise statements about the requirements that should be placed on the scalar potential couplings to obtain the desired phase transition. Therefore, we simply use the arguments presented and focus on triplets with negative quadratic terms $-\mu_{\Sigma}^{2}<0$ and, as a consequence, positive Higgs couplings $a_{2}>0$. One potential caveat is that for models with further extensions to the scalar sector, it is possible that some other particle (e.g. a scalar singlet) may have gained a VEV that acts to destabilise the triplet in the early universe, or may have a VEV at zerotemperature acting to increase the mass of the triplet [4]. This allows for the possibility that $-\mu_{\Sigma}^{2}>0$ while still letting the triplet gain a VEV in the early universe. Hence, we will also examine the parameter space where $-\mu_{\Sigma}^{2}$ takes on small positive values $-\mu_{\Sigma}^{2} \sim(100 \mathrm{GeV})^{2}$, despite the fact that such further extensions might significantly affect the phenomenology.

Combining the requirement that $\mu_{\Sigma}^{2}>0$ with the requirement that the scalar couplings satisfy perturbativity and perturbative unitarity then directly leads to an upper bound on the mass of the triplet. From figure 1, we see that requiring perturbativity up to $\Lambda=1 \mathrm{TeV}$ requires $m_{h_{2}} \lesssim 415 \mathrm{GeV}$. If we instead require perturbativity up to $10^{6} \mathrm{GeV}$, this upper bound decreases to $m_{h_{2}} \lesssim 270 \mathrm{GeV}$.

\subsection{Higgs diphoton rate}

In the SM the Higgs can decay into two photons via a fermion or $W^{ \pm}$loop. The introduction of the triplet scalar will lead to a correction to the SM Higgs diphoton rate via the addition of a new charged scalar loop. This correction is proportional to $a_{2}$ and decreases with increasing charged scalar mass. However, in our scenario a larger mass necessarily means a larger $a_{2}$, and hence a precise measurement of the diphoton rate could in principle be used to exclude triplets with negative quadratic coefficients altogether. The SM diphoton rate is given by [42]

$$
\Gamma_{H \rightarrow \gamma \gamma}^{\mathrm{SM}}=\frac{\alpha^{2} g_{2}^{2}}{1024 \pi^{3}} \frac{m_{H}^{3}}{m_{W}^{2}}\left|\frac{4}{3} F_{1 / 2}\left(4 \frac{m_{t}^{2}}{m_{H}^{2}}\right)+F_{1}\left(4 \frac{m_{W}^{2}}{m_{H}^{2}}\right)\right|^{2} .
$$

\footnotetext{
${ }^{3}$ The feature of the third extremum that $v_{\Sigma}=0$ at zero temperature then requires that the $a_{2} v_{H}^{2}$ induced contribution be sufficiently large so that the effective quadratic coefficient $-\mu_{\Sigma}^{2}+\frac{1}{2} a_{2} v_{H}^{2}$ is both positive and large enough to produce phenomenologically-viable triplet scalar masses. In this situation, $a_{2}$ must be positive.
} 
Neglecting the small charged scalar mixing angle $\theta_{C}$, the triplet modifies the diphoton rate to $[6]$,

$$
\begin{aligned}
\Gamma_{h_{1} \rightarrow \gamma \gamma}^{\Sigma \mathrm{SM}} \approx \frac{\alpha^{2} g_{2}^{2}}{1024 \pi^{3}} \frac{m_{h_{1}}^{3}}{m_{W}^{2}} & \frac{4}{3} \cos \theta_{N} F_{1 / 2}\left(4 \frac{m_{t}^{2}}{m_{h_{1}}^{2}}\right) \\
& +\left(\cos \theta_{N}-\sin \theta_{N} \frac{4 v_{\Sigma}}{v_{H}}\right) F_{1}\left(4 \frac{m_{W}^{2}}{m_{h_{1}}^{2}}\right) \\
& +\left.\left(\cos \theta_{N} a_{2}-\sin \theta_{N} b_{4} \frac{2 v_{\Sigma}}{v_{H}}\right) \frac{v_{H}^{2}}{2 m_{h^{+}}^{2}} F_{0}\left(4 \frac{m_{h^{+}}^{2}}{m_{h_{1}}^{2}}\right)\right|^{2},
\end{aligned}
$$

where the loop functions are,

$$
\begin{aligned}
F_{0}(x) & =x(1-x f(x)) \\
F_{1 / 2}(x) & =-2 x(1+(1-x) f(x)) \\
F_{1}(x) & =2+3 x(1+(2-x) f(x)) \\
f(x) & =\left\{\begin{array}{ll}
\arcsin ^{2}(1 / \sqrt{x}) & x \geq 1 \\
-\frac{1}{4}\left(\ln \frac{1+\sqrt{1-x}}{1-\sqrt{1-x}}-i \pi\right)^{2} & x<1
\end{array} .\right.
\end{aligned}
$$

The $\mathbb{Z}_{2}$ symmetric result can be obtained by setting $\theta_{N}$ and $v_{\Sigma}$ to zero. The scalar consisting primarily of the triplet can also decay into two photons, with rate given by

$$
\begin{aligned}
\Gamma_{h_{2} \rightarrow \gamma \gamma}^{\Sigma \mathrm{SM}} \approx \frac{\alpha^{2} g_{2}^{2}}{1024 \pi^{3}} \frac{m_{h_{2}}^{3}}{m_{W}^{2}} & \mid \frac{4}{3} \sin \theta_{N} F_{1 / 2}\left(4 \frac{m_{t}^{2}}{m_{h_{2}}^{2}}\right) \\
+ & \left(\sin \theta_{N}-\cos \theta_{N} \frac{4 v_{\Sigma}}{v_{H}}\right) F_{1}\left(4 \frac{m_{W}^{2}}{m_{h_{2}}^{2}}\right) \\
& +\left.\left(\sin \theta_{N} a_{2}+\cos \theta_{N} b_{4} \frac{2 v_{\Sigma}}{v_{H}}\right) \frac{v_{H}^{2}}{2 m_{h^{+}}^{2}} F_{0}\left(4 \frac{m_{h^{+}}^{2}}{m_{h_{2}}^{2}}\right)\right|^{2}
\end{aligned}
$$

The signal strength of the SM Higgs to diphoton process is then given by

$$
\mu_{\gamma \gamma}=\frac{\Gamma_{h_{1} \rightarrow \gamma \gamma}^{\Sigma \mathrm{SM}} .}{\Gamma_{H \rightarrow \gamma \gamma}^{\mathrm{SM}} .}
$$

We compare this with the most recent measurements by the ATLAS [43] and CMS [44] collaborations,

$$
\mu_{\gamma \gamma}^{\mathrm{ATLAS}}=0.99 \pm 0.14, \quad \mu_{\gamma \gamma}^{\mathrm{CMS}}=1.18_{-0.14}^{+0.17} .
$$

We combine these measurements using a simple inverse variance weighted average. Taking $0.14^{2}$ to be the variance of the CMS measurement this yields,

$$
\mu_{\gamma \gamma}^{\text {expt }}=1.085 \pm 0.099 .
$$

Figure 2 shows the contour plots of the SM Higgs diphoton signal strength as a function of $m_{h_{2}}$ and either $\mu_{\Sigma}^{2}$ or $a_{2}$ in the $\mathbb{Z}_{2}$ symmetric case. The $\mathbb{Z}_{2}$ broken case only differs significantly near $m_{h_{2}} \approx m_{h_{1}}$, where the triplet-Higgs mixing angles are large. 

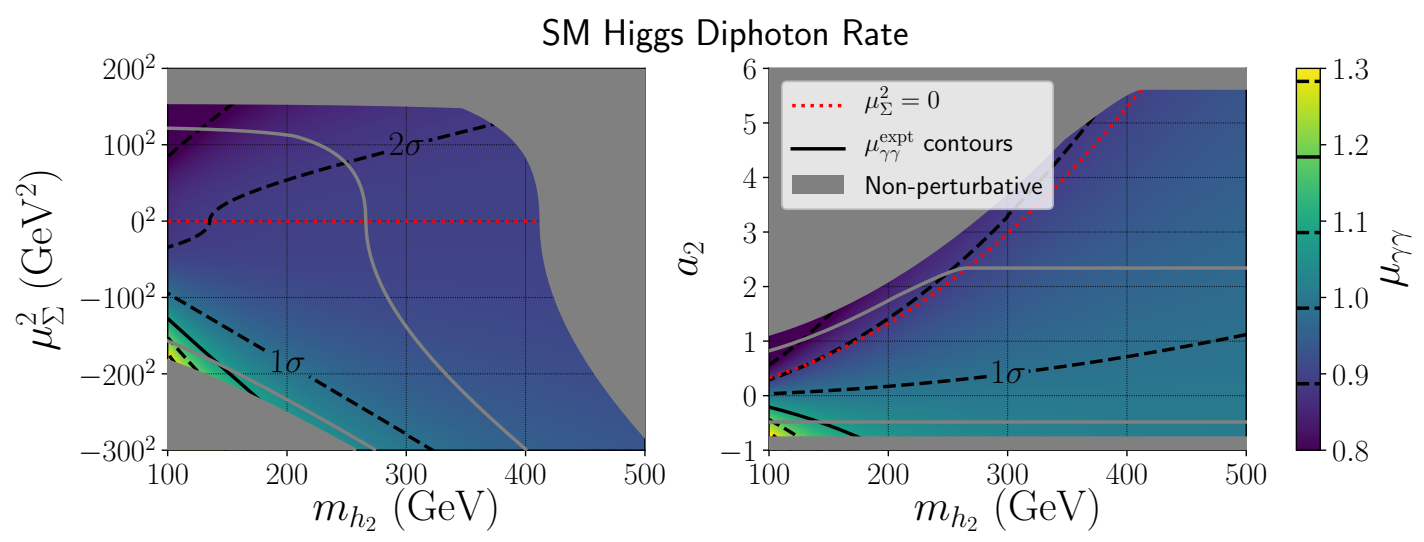

Figure 2. SM Higgs diphoton rate as a function of the triplet-like neutral scalar mass $m_{h_{2}}$ and either $\mu_{\Sigma}^{2}$ (left) or $a_{2}$ (right), with $v_{\Sigma}=0$ and $b_{4}=1$. The solid black line is the contour of the combined CMS and ATLAS diphoton rate measurement, and the dashed lines give the one-, two- and three-sigma contours. The solid grey region is the parameter-space where the scalar couplings become non-perturbative at energies $\Lambda<1 \mathrm{TeV}$, and the solid grey line shows where this contour would be if the cutoff energy is increased to $\Lambda=10^{6} \mathrm{GeV}$. The red dotted line indicates the $\mu_{\Sigma}^{2}=0$ contour.

The future prospects for measuring the SM Higgs diphoton signal strength at the HighLuminosity LHC indicate an expected error of $\sim 10 \%$ with $3 \mathrm{ab}^{-1}$ of data at $14 \mathrm{TeV}[45,46]$. Assuming the measured value moves towards the SM prediction $\mu_{\gamma \gamma}=1$, this enhanced accuracy will not result in constraints that are significantly more stringent than the current ones, as the shift towards a SM value would offset the decrease in error.

\section{$3 \quad$ Stable triplet phenomenology}

\subsection{Disappearing tracks}

As discussed in section 2.1, in the $\mathbb{Z}_{2}$ symmetric model the small radiative mass splitting allows the charged triplet component to decay via an off-shell $W^{ \pm}$into a neutral triplet component and a low energy pion or lepton pair. As the triplet mass varies from $100 \mathrm{GeV}$ to $1 \mathrm{TeV}$, the lifetime varies between $0.1-0.18 \mathrm{~ns}(c \tau=3-5 \mathrm{~cm})$. Hence, as pointed out by refs. $[18,22]$ charged triplets may result in disappearing charged tracks at the LHC. Recent searches for disappearing tracks produced by decaying charginos were performed by the CMS [47] and ATLAS [48] collaborations using $36 \mathrm{fb}^{-1}$ of data. The ATLAS disappearing track searches are more sensitive to small lifetimes than the CMS searches. As the triplets will have small lifetimes, the ATLAS searches provide the most severe constraints. The ATLAS analysis provides a model-independent 95\% confidence upper bound on the visible cross section, alongside efficiency times acceptance data for the production of charginos as a function of their lifetime and mass [48, 49]. One of the production mechanisms considered in the ATLAS analysis is pair-production of charginos via charged or neutral current Drell-Yan processes, with cuts applied to the initial state radiation jets and disappearing charged tracks. Charged and neutral current Drell-Yan processes are also the dominant pair production processes for the charged triplets. Hence, we directly take the chargino 
acceptance times efficiency data, linearly interpolate it, and apply it to the charged triplet production cross section. Combining this with the model-independent $95 \%$ confidence upper bound on the visible cross section then yields an upper bound on the charged triplet production cross section. Note that the production of the charged triplet components (scalars) will lead to disappearing track $p_{T}$ and $\eta$ distributions that differ from those in chargino production (fermions). Similarly, the leading jet $p_{T}$ will also differ. However, the charged scalar production distributions are skewed towards higher $p_{T}$ and lower $|\eta|$ values, such that the acceptance times efficiency for charged triplet production is likely higher than for chargino production. Thus using the chargino acceptance times efficiency data should result in a conservative estimate for the disappearing track bound. The triplets may also be pair produced via an intermediate SM Higgs boson produced, increasing the total production cross section. However this production process will likely have a different jet distribution, such that the given acceptances and efficiencies likely do not apply. We will set $\mu_{\Sigma}^{2}=-m_{h_{2}}^{2}\left(a_{2}=0\right)$, and ignore this production process in this section.

To interpret the interpolated disappearing track search results, we need the lifetime and production cross section for the charged triplets. The lifetimes were calculated using eqs. (2.9) and (2.11). We utilise MadGraph5_aMC@NLO 2.6.5 [50] to evaluate the production cross section at NLO, using an NLO compatible UFO [51] model file generated using FeynRules 2.3.32 [52], FeynArts 3.9 [53, 54], and NLOCT 1.02 [55]. The charged triplet lifetime and production cross sections are then only dependent on the mass of the triplet, and the disappearing track searches can be used to place a lower bound on that mass.

The resulting cross sections, interpolated limit, and lifetimes are shown in figure 3 . The cross section drops below the interpolated limit for masses $m_{h_{2}} \gtrsim 250 \mathrm{GeV}$, and we take this to be the lower bound on stable triplets arising from disappearing tracks. While LEP has searched for displaced vertices in the context of SUSY searches for chargino pair-production, due to the smaller cross-section for scalar production and threshold effects, the limits from these searches for scalars are likely to be less than the $100 \mathrm{GeV}$ usually stated $[56,57]$.

This bound is very sensitive to the lifetime of the charged triplet component, which itself depends on the mass splitting of the charged and neutral components of the triplet. The lifetime of fermionic multiplets decaying due to radiative mass splitting has been found to change significantly when performing a two-loop mass splitting calculation $[58,59]$. In the fermionic case, the mass splitting decreases and the lifetime goes up, which is favourable for the reach of disappearing track searches. Reliably excluding the triplet would require a precise calculation of the scalar two-loop radiative mass splitting, which is beyond the scope of our analysis. Additionally, note that the lifetime of the charged triplet decreases with decreasing mass. This is a result of the fact that the one-loop radiative mass splitting, eq. (2.9), is larger for smaller triplet masses. ${ }^{4}$ Thus, for some mass less than $100 \mathrm{GeV}$ the lifetime will be too short to leave disappearing tracks, and will not be constrained by these analyses. As the available ATLAS disappearing track data only goes down to chargino masses of about $100 \mathrm{GeV}$, it is not clear at what mass the decrease in lifetime overpowers the increasing production cross section.

\footnotetext{
${ }^{4}$ This is not the case for fermionic multiplets. The fermionic mass splitting is smaller for smaller masses, such that the lifetime increases.
} 


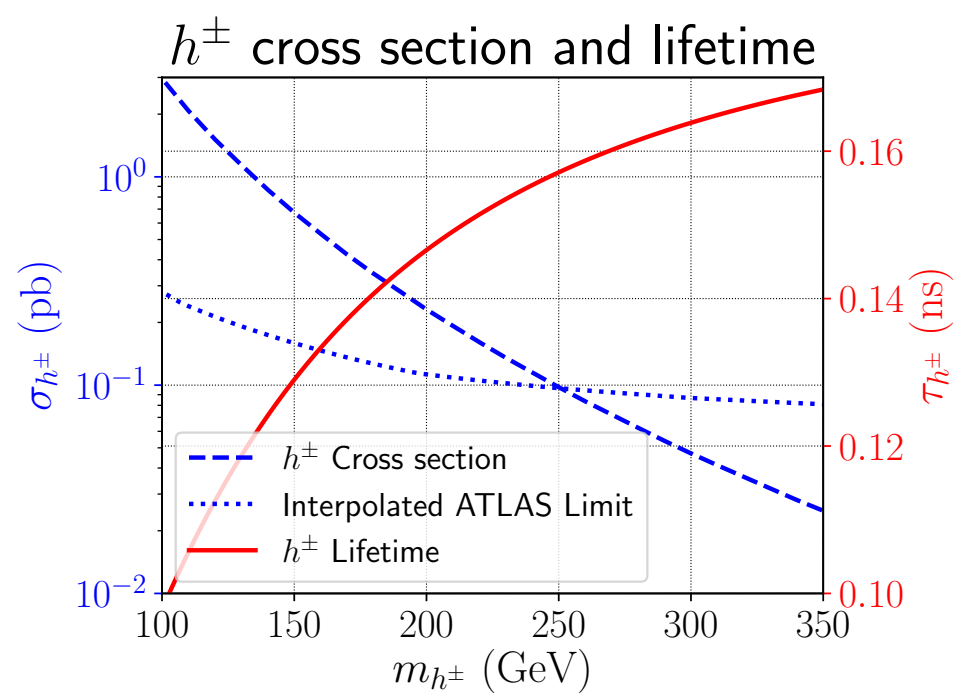

Figure 3. Charged triplet production cross section (dashed blue line) and lifetime (solid red line), along with the interpolated $95 \%$ confidence upper limit on the chargino production cross section (dotted blue line) arising from disappearing tracks searches for charginos with the same mass and lifetime. The charged triplet production cross section intersects the upper limit at $m_{h^{+}} \approx 250 \mathrm{GeV}$, and we take this to be the lower bound imposed by disappearing track searches.

\subsection{Dark matter direct detection}

The real SU(2) triplet scalar thermal dark matter model has been studied extensively [2228]. The annihilation into weak gauge bosons requires that the triplet have a mass $m_{h_{2}} \sim 2 \mathrm{TeV}$ in order to obtain the right relic density. Inclusion of annihilation via the SM Higgs necessitates an even larger mass. Hence a triplet with $m_{h_{2}} \lesssim 500 \mathrm{GeV}$, as required by our constraints, will only ever constitute a small fraction of the relic density. However, if we require $\mu_{\Sigma}^{2}>0$, the triplet will have a large coupling to the SM Higgs. This coupling provides the dominant contribution to the nuclear scattering cross section, and thus constrains the triplet even for very small relic abundances.

In order to investigate this bound in more detail we utilise MicrOMEGAS 5.0.8 [60] to evaluate the triplet relic abundance. We normalise the relic abundance by the dark matter density measured by the Planck collaboration [61], $\Omega_{\mathrm{DM}} h^{2}=0.12$. The MicrOMEGAS results were verified by comparison with results obtained using MadDM 3.0 [62] and they were found to be in good agreement. However, it is important to note that neither MicrOMEGAS nor MadDM include the Sommerfeld enhancement. The Sommerfeld enhancement arises due to the attractive potential between two DM particles resulting in an increase in the DM annihilation rate, with a corresponding decrease in the relic density. The effect is suppressed if the electroweak symmetry is broken and the weak gauge bosons gain masses comparable to the DM mass. Given that freeze-out typically occurs at temperatures $T_{f} \sim m_{\mathrm{DM}} / 25$ and as we are interested in triplets with $m_{h_{2}}<500 \mathrm{GeV}$, which implies $T_{f} \lesssim 20 \mathrm{GeV}$, we expect the electroweak symmetry to have been broken by the time the triplets freeze out. However, even with massive gauge bosons, the Sommerfeld effect can still reduce the relic density by 

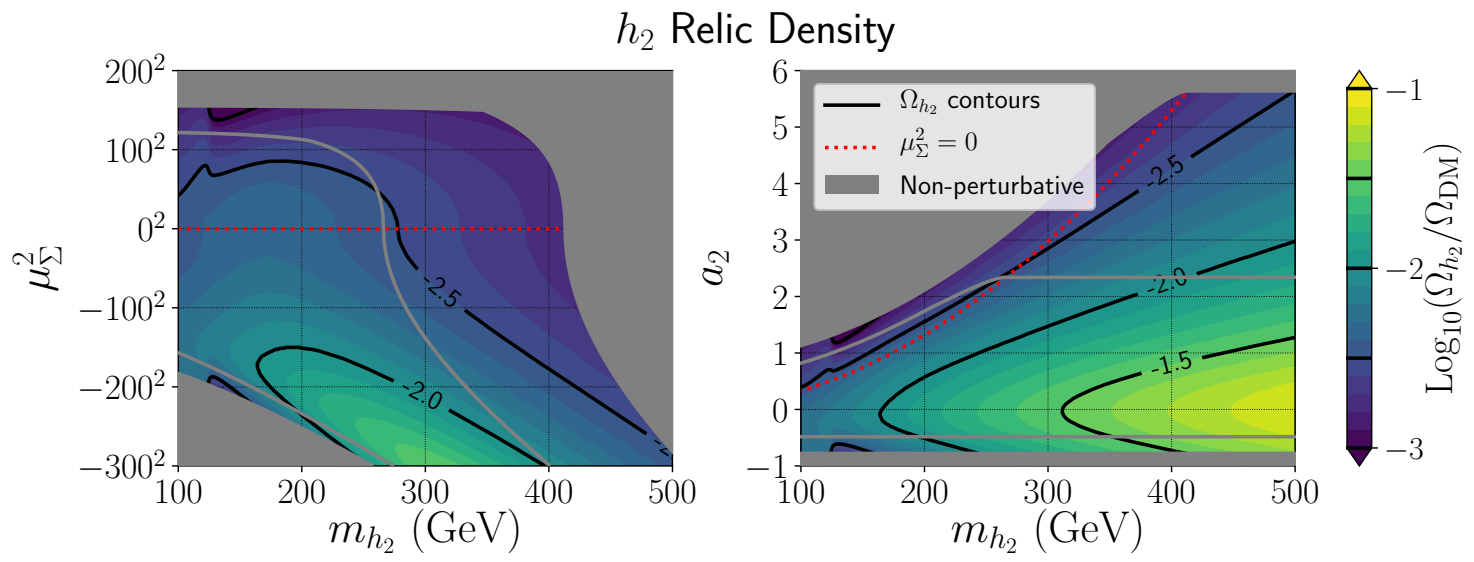

Figure 4. The log of triplet dark matter relic density as a function of $m_{h_{2}}$ and either $\mu_{\Sigma}^{2}$ (left) or $a_{2}$ (right), normalised to the observed value from Planck. The solid grey region is the parameter-space where the scalar couplings become non-perturbative at energies $\Lambda<1 \mathrm{TeV}$, and the solid grey line shows where this contour would be if the cutoff energy is increased to $\Lambda=10^{6} \mathrm{GeV}$. As a function of $a_{2}$, the relic density reaches a maximum at $a_{2}=0\left(\mu_{\Sigma}^{2}=-m_{h_{2}}^{2}\right)$, such that the $h_{2} h_{2} \rightarrow h_{1} h_{1}$ annihilation rate is zero. The red dotted line is the $\mu_{\Sigma}^{2}=0$ contour.

15-30\% for triplets with masses $m_{h_{2}}=400-1000 \mathrm{GeV}$ [23]. We will not perform a rigorous calculation accounting for the Sommerfeld enhancement and will simply note that there is a $\sim 15 \%$ uncertainty on the relic density and resulting DM detection exclusion plots.

In addition to neglecting the Sommerfeld enhancement, we also ignore bound state effects as they are negligible for the parameter-space that we consider. Furthermore, we also utilise the zero-temperature mass for the triplet during the relic density calculation. If the triplet's mass at zero temperature arises primarily through the Higgs VEV, its mass may change significantly in the early universe. However, as we expect freeze-out to occur at $T_{f} \lesssim 20 \mathrm{GeV}$, we expect $v_{H}, m_{h_{2}}$, and $m_{h_{2}}$ to be close to their zero temperature values, such that this is a minor correction. This approximation is motivated by noting that in the $\mathrm{SM}$, there is a crossover transition at $T_{c} \sim 160 \mathrm{GeV}$ [63], with the SM Higgs VEV approximately decreasing as $v_{H}(T) \sim v_{H}(0) \sqrt{1-\frac{T^{2}}{T_{c}^{2}}}$. Thus at freeze-out one might reasonably expect $v_{H}\left(T_{f}\right) / v_{H}(0)=0.99$, such that using the zero-temperature value for the Higgs VEV at $T \lesssim 20 \mathrm{GeV}$ is a reasonable approximation in the SM. We assume this approximation remains reasonable despite changes to the electroweak phase transition due the addition of the triplet. A precise determination of the relic density would require a proper calculation for the phase transition for each parameter point in order to obtain the correct temperature dependent masses.

The resulting relic densities are shown in figure 4 as a function of $m_{h_{2}}$ and either $\mu_{\Sigma}^{2}$ or $a_{2}$. Unless $m_{h_{2}} \gtrsim 500 \mathrm{GeV}$ and $a_{2} \sim 0\left(\mu_{\Sigma}^{2} \sim-m_{h_{2}}^{2}\right)$, such that the annihilation rate into two SM Higgs bosons is small, the neutral triplet makes up less than $10 \%$ of the total dark matter density. The slight jump in relic density for $m_{h_{2}}<125 \mathrm{GeV}$ occurs due to the kinematic suppression of the $h_{2} h_{2} \rightarrow h_{1} h_{1}$ annihilation channel, leading to a larger relic density. 

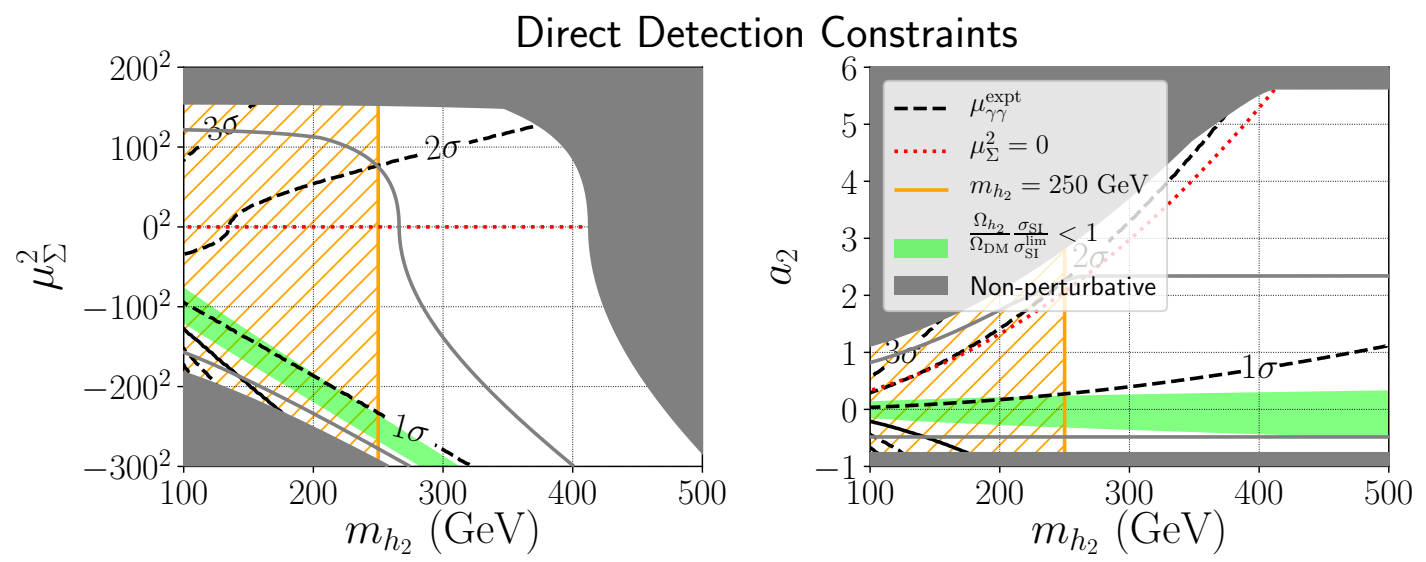

Figure 5. The region of parameter space consistent with the current XENON1T direct detection limit as a function of $m_{h_{2}}$ and either $\mu_{\Sigma}^{2}$ (left) or $a_{2}$ (right). The allowed parameter-space is shown as a green band. The solid grey region is the parameter-space where the scalar couplings become non-perturbative at energies $\Lambda<1 \mathrm{TeV}$, and the solid grey line shows where this contour would be if the cutoff energy is increased to $\Lambda=10^{6} \mathrm{GeV}$. The black lines indicate the diphoton rate contours as in figure 2. The hashed orange region is the lower bound on $m_{h_{2}}$ from disappearing tracks that was obtained in section 3.1. The red dotted line is the $\mu_{\Sigma}^{2}=0$ contour.

The spin-independent (SI) nuclear scattering cross section $\sigma_{\mathrm{SI}}$ is then obtained using the formulae given in ref. [64], which takes into account the one-loop scattering cross section generated by $W^{ \pm}$box-diagrams. The cross section is then compared to the XENON1T [65] $90 \%$-confidence upper bound on the SI scattering cross section $\sigma_{\mathrm{SI}}^{\lim }$, after scaling to account for the fraction of the density of DM that is made up of $h_{2}$. Figure 5 shows the constraints from the XENON1T experiment, along with the lower bound imposed by disappearing track searches. A stable triplet with $\mu_{\Sigma}^{2}>0$ is ruled out by dark matter direct detection constraints. The only region allowed is a strip where $\mu_{\Sigma}^{2} \sim-m_{h_{2}}^{2}$, corresponding to $\left|a_{2}\right| \lesssim$ 0.5, where the triplet coupling to the SM Higgs is small. This is shown as a green band in figure 5. As the rate for DM self-annihilation is proportional to the number density squared, the annihilation rate is very low for these relic densities. Hence, there are no constraints from dark matter indirect detection experiments. Inclusion of the Sommerfeld enhancement would result in a slightly larger allowed region.

Higher representation SU(2) multiplets are also strongly constrained by dark matter direct detection constraints, forcing the coupling between the SM Higgs and scalar electroweak multiplet dark matter to be small [5, 64]. These direct detection constraints are not always applied even when they rule out a significant region of the benchmark points considered, as is the case for refs. [3, 6, 17]. These models then require either allowing for $\mu_{\Sigma}^{2}<0$ and tuning the DM-Higgs coupling to be small, which is unfavourable for two step phase transition models, or breaking the $\mathbb{Z}_{2}$ which stabilises the DM, so that it is not a DM candidate any more. ${ }^{5}$ Breaking the symmetry is straightforward in the case of the scalar triplet. No additional particle content is needed as allowing a non-zero $a_{1}$ coupling

\footnotetext{
${ }^{5}$ If the scalars are long lived but unstable, the disappearing track constraints will still apply.
} 


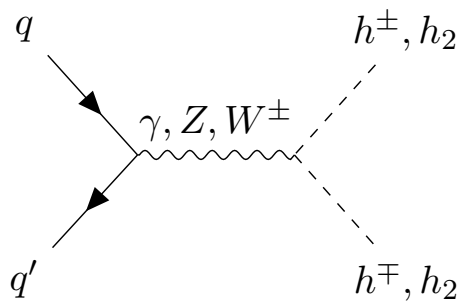

(a)

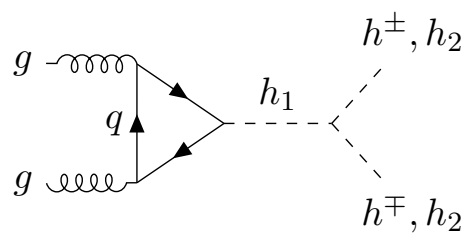

(b)

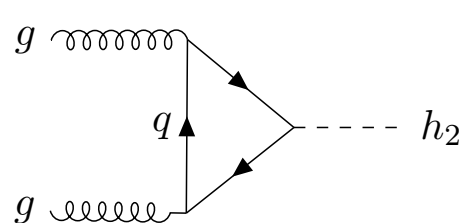

(c)

Figure 6. Feynman diagrams showing the primary production processes for the new scalars, including Drell-Yan pair production (a), pair production via an intermediate off-shell $h_{1}$ (b), and single $h_{2}$ production via $g g \mathrm{~F}(\mathrm{c})$.

breaks the symmetry. This $\mathbb{Z}_{2}$ breaking coupling can be very small, such that it will not significantly change the results of phase transition studies. However, one must then contend with new constraints arising from other collider searches, and it is to this possibility that we now turn our attention.

\section{Unstable triplet phenomenology}

\subsection{Production processes}

The primary production processes for the SM Higgs boson at the LHC are via gluon-gluon fusion $(g g \mathrm{~F})$ and vector-boson fusion (VBF). However, neither of these processes will lead to appreciable $h_{2}$ production. This is due to the fact that the coupling to the heavy quarks involved in $g g \mathrm{~F}$ is suppressed by a factor of $\sin \theta_{N}$, leading to a significantly smaller production rate. Additionally, the $W W h_{2}$ and $Z Z h_{2}$ vertices necessary for VBF arise due to neutral scalar mixing (suppressed by $\sin \theta_{N}$ ) or via the triplet's VEV (suppressed by $\left.v_{\Sigma} / v_{H}\right)$. Other SM-Higgs production mechanisms are similarly suppressed. Hence, unless $h_{1}$ and $h_{2}$ are nearly degenerate, such that there is a sizeable mixing angle, single $h_{2}$ production will be several orders of magnitude smaller than SM Higgs production cross sections. Single $h^{ \pm}$production will similarly be suppressed by factors of $\sin \theta_{C}$ and $v_{\Sigma} / v_{H}$. As a result, the primary production mechanism for the new scalars is via neutral or charged current Drell-Yan pair production. Additionally, pair production via an intermediate offshell SM Higgs may contribute significantly. In the SM, Higgs pair production is suppressed due to the small cubic coupling $\lambda_{H} v_{H}$, and due to the interference of the box and triangle diagrams [66]. However, in our scenario the coupling $a_{2} v_{H}$ may be large and the interfering box diagram is suppressed by a factor of $\sin ^{2} \theta_{N}$. Thus production via an off shell $h_{1}$ can form a significant contribution for large $a_{2}$. We will therefore include pair production via an off-shell intermediate $h_{1}$ produced through $g g \mathrm{~F}$. All $h_{1}$-style production processes will contribute in such a manner. However, as $g g \mathrm{~F}$ is the dominant production process for single $h_{1}$ and as Drell-Yan pair production dominates anyway, neglecting other off-shell $h_{1}$ pair production diagrams will have no significant effect on the results. Feynman diagrams for the dominant production processes are shown in figure 6 . 


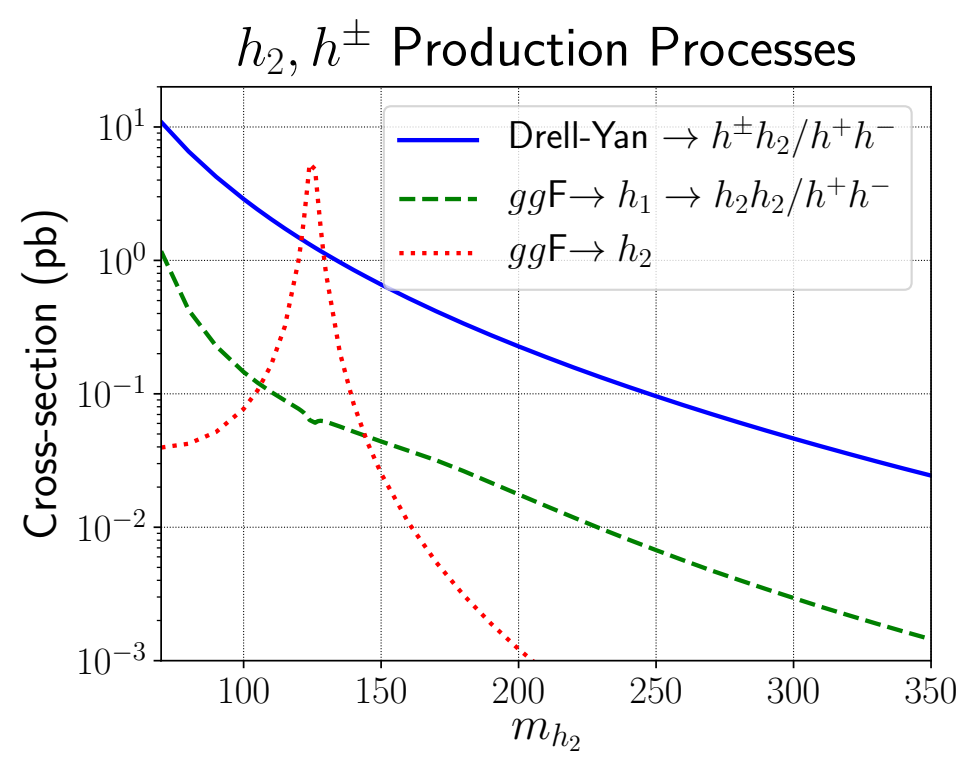

Figure 7. The total $h_{2}, h^{ \pm}$pair production cross sections via Drell-Yan processes (solid blue line) or via an intermediate off-shell $h_{1}$ (green dashed line), as well as the single $h_{2}$ production cross section via $g g \mathrm{~F}$ (dotted red line). The cross sections are shown for $\mu_{\Sigma}^{2}=200^{2} \mathrm{GeV}^{2}$ and $v_{\Sigma}=1$. The peak in the single $h_{2}$ production cross at $m_{h_{2}} \approx 125 \mathrm{GeV}$ occurs due to an increase in the neutral scalar mixing angle when the two neutral scalars are nearly degenerate, and not due to an $s$-channel resonance.

Figure 7 shows the pair production cross sections for the new scalars via Drell-Yan or via an intermediate off-shell $h_{1}$, in addition to single $h_{2}$ production via $g g \mathrm{~F}$. The cross sections were obtained using MadGraph5. The Drell-Yan cross section was evaluated with NLO QCD corrections, while the off-shell $h_{1}$ and $g g \mathrm{~F} h_{2}$ production cross sections are loop induced processes evaluated at leading order. As argued earlier, the cross section for the production of a single $h_{2}$ is via $g g \mathrm{~F}$ is suppressed by $\sin \theta_{N}$, such that it is large only when $m_{h_{1}} \approx m_{h_{2}}$. Pair production dominates away from this region, and will always dominate if $v_{\Sigma} \lesssim 0.5 \mathrm{GeV}$. Furthermore, pair produced $h_{2}$ lead to multi-gauge boson events with significantly smaller backgrounds, and as a result we focus on pair production at colliders.

Note that $g g \mathrm{~F}$ Higgs production increases significantly with the inclusion of higher order corrections, with a $k$-factor of around 3 at $\mathrm{N}^{3} \mathrm{LO}$ [67]. The cross sections shown in figure 7 are unmodified. Even with the correction, single $h_{2}$ production remains subdominant for most of the parameter-space. However, this raises a concern that the higher order corrections to the pair production through intermediate off-shell $h_{1}$ are similarly significant. Naively, as the QCD component of the $g g \mathrm{~F}$ single Higgs and new scalar $g g \mathrm{~F}$ pair production are the same, one might expect a $k$-factor of $k \sim 3$. In contrast, the $k$-factor for SM Higgs pair production is $k \sim 2$ [68-70]. As mentioned before, this process is different as it features an additional interfering box diagram. It is unclear which $k$-factor is more readily applicable to the new scalar $g g \mathrm{~F}$ pair production process. We take the lower of the two and scale this cross section by a $k$-factor of $k=2$. With the $k$-factor correction, pair production via an intermediate $h_{1}$ results in a $10-20 \%$ increase in the overall pair 
production cross section. Furthermore, it is the only source of $h_{2} h_{2}$ pairs, as they are not produced via neutral-current Drell-Yan processes.

\subsection{Decay channels}

The $h_{2}$ and $h^{ \pm}$scalars have three means of decaying:

- Decay via mixing with the SM Higgs or charged Goldstone into fermions and gauge bosons. For the $h_{2}$, these partial widths are suppressed relative to SM Higgs decays by a factor of $\sin \theta_{N}$, while the partial widths for the $h^{ \pm}$will be proportional to $\sin \theta_{C}$.

- Decay via $v_{\Sigma}$ into weak gauge bosons $\left(W^{ \pm} W^{\mp}, Z Z, W^{ \pm} Z\right)$. These partial widths are suppressed by $v_{\Sigma} / v_{H}$ relative to similar SM Higgs decays.

- Decay into $h_{1} h_{1}$ or $W^{ \pm} h_{1}$. These partial widths are proportional to $v_{\Sigma}+v_{H} \sin \theta_{N}$ and $\sin \theta_{C}+2 \sin \theta_{N}$, respectively.

Thus, aside from the $h^{ \pm} \rightarrow h_{1} W^{ \pm}$channel which depends on $\theta_{N}$, the partial widths of the charged scalar are completely determined by its mass (kinematics) and $v_{\Sigma}$ (which fixes $\theta_{C}$ ). The scenario for the neutral scalar is more complicated, as $\theta_{N}$ is a function of $v_{\Sigma}, \mu_{\Sigma}^{2}$ and $m_{h_{2}}$. Additionally, one must include the $b_{4}$ dependent diphoton rate. For the purposes of the decay phenomenology, changing $\mu_{\Sigma}^{2}$ affects the size of the neutral scalar mixing angle $\theta_{N}$. In particular, note that from eq. (2.13), if $\mu_{\Sigma}^{2}=v_{\Sigma}^{2} b_{4}$, then $v_{\Sigma}=\frac{a_{1}}{2 a_{2}}$ such that the off-diagonal term in the scalar mass mixing matrix disappears and we get $\theta_{N}=0$. Hence when $\mu_{\Sigma}^{2}$ is small, of the order of a few $\mathrm{GeV}^{2}$, the neutral scalar mixing angle $\theta_{N}$ will also be very small and the decays of the neutral triplet will be dominated by decays into weak gauge bosons $h_{2} \rightarrow W^{ \pm} W^{\mp(*)}$. Conversely, a larger $\left|\mu_{\Sigma}^{2}\right|$ corresponds to a larger $\theta_{N}$. Finally, as both $\theta_{N}$ and $\theta_{C}$ are both proportional to $v_{\Sigma}$, the triplet VEV sets the overall size of the widths and has very little impact on the branching fractions.

To obtain the partial widths for decays into fermions and gluons arising from mixing with the SM Higgs, we utilise the HDECAY 6.511 [71] package. The $h_{2}$ partial widths for decays into fermions and gluons are those of a SM Higgs of mass $m_{h_{2}}$ scaled by $\sin ^{2} \theta_{N}$. For the diphoton rate $\Gamma_{h_{2} \rightarrow \gamma \gamma}^{\Sigma \mathrm{SM}}$ we use the analytic formulae given in eq. (2.27). We do not include the $h_{2} \rightarrow Z \gamma$ decay. The partial widths for the decay of $h^{ \pm}$into fermions are obtained from the partial widths of a charged Higgs in a type-I $2 \mathrm{HDM}$ with $\tan \beta=\frac{1}{\tan \theta_{C}}$ and $\sin (\alpha)=0$, as given by HDECAY. The other decays into scalars and electroweak gauge bosons were obtained automatically by MadWidth [72], a component of MadGraph5.

The resulting branching fractions and partial widths are shown in figures 8 and 9 for the triplet-like neutral and charged scalar, respectively. The $h_{2}$ width features a resonance at $m_{h_{2}} \approx 125 \mathrm{GeV}$ due to a large neutral scalar mixing angle $\theta_{N}$. However, the branching fractions are relatively smooth and instead feature a transition between fermionic and electroweak decays due to kinematic suppression. For small masses, the $h_{2}$ decay primarily into $b \bar{b}, \tau^{+} \tau^{-}$, and $c \bar{c}$. Conversely, for larger masses they decay primarily into $W^{ \pm} W^{\mp}$ and $h_{1} h_{1}$, with the branching ratio into the latter being strongly dependent on $\mu_{\Sigma}^{2}$. The charged scalar will decay mostly into $\tau \nu$ or $c s$ fermions if $m_{h^{ \pm}} \lesssim 120 \mathrm{GeV}$, and into $t b, W^{ \pm} h_{1}$, or 

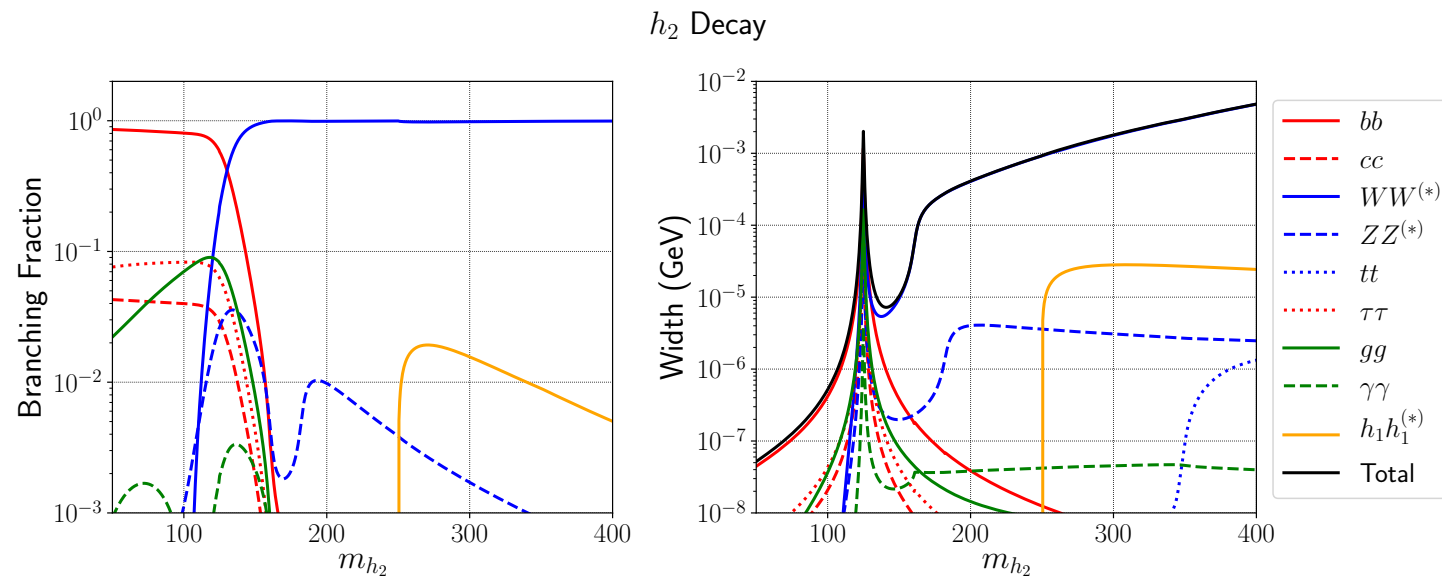

Figure 8. Branching fractions (left panel) and partial widths (right panel) for the decay of $h_{2}$, for $\mu_{\Sigma}^{2}=100^{2} \mathrm{GeV}^{2}, v_{\Sigma}=1 \mathrm{GeV}$, and $b_{4}=1$. The peak at $m_{h_{2}} \approx 125 \mathrm{GeV}$ occurs due to an increase in the neutral scalar mixing angle when the two neutral scalars are nearly degenerate.
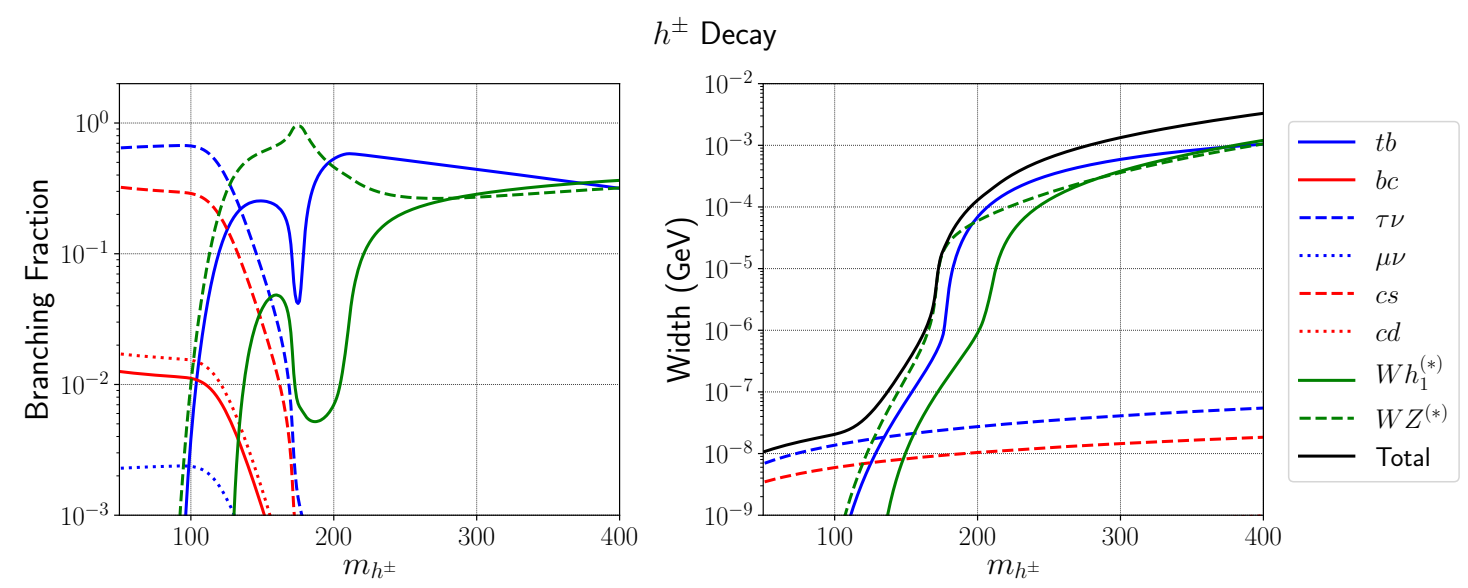

Figure 9. Branching fractions (left panel) and partial widths (right panel) for the decay of $h^{+}$for $\mu_{\Sigma}^{2}=100^{2} \mathrm{GeV}^{2}, v_{\Sigma}=1 \mathrm{GeV}$, and $b_{4}=1$.

$W^{ \pm} Z$ pairs when heavier. Note that if we had fixed $a_{2}$ instead of setting $\mu_{\Sigma}^{2}=100^{2} \mathrm{GeV}^{2}$ the behaviour of the branching fraction would be significantly different. In particular, if $a_{2}$ is negative, then $\operatorname{Br}\left(h_{2} \rightarrow Z Z\right)$ can become large. Additionally, if $\mu_{\Sigma}^{2} \sim v_{\Sigma}^{2} b_{4}$ such that $\theta_{N}$ is small and the SM Higgs mixing-induced decays into two fermions are suppressed, and $m_{h_{2}} \lesssim m_{W}$, so that the decays into weak gauge bosons are kinematically suppressed, then the $h_{2}$ diphoton branching fraction can become significant.

Note that our choice to fix the cubic term $a_{1}$ as a function of $v_{\Sigma}$ significantly affects the behaviour of the widths as a function of mass. If we had instead selected a value for $a_{1}$ and used that to fix $v_{\Sigma}$ (leading to $v_{\Sigma} \propto 1 / m_{h_{2}}^{2}$ ), the partial widths would decrease as the mass of the triplet becomes very large. However, as mentioned earlier, varying $v_{\Sigma}$ scales the overall widths without affecting the branching ratios. Hence, the phenomenological results would be the same. 


\subsection{Collider searches}

There are a range of ATLAS and CMS analyses searching for exotic scalars. However, these searches generally focus on single scalar production. Examples include searches for new neutral scalars decaying into $\gamma \gamma[73-76]$ or $\tau^{+} \tau^{-}$[77], and searches for new charged scalars decaying into $\tau \nu_{\tau}[78,79], t b[80,81]$, or $W Z[82,83]$. In our scenario the dominant source of new scalars is via pair production. While pair production may lead to signal events in these searches, dedicated pair production searches would have significantly lower backgrounds, and thus, would be significantly more constraining. Taking the constraints on the production cross section times branching fractions obtained by these analyses and directly interpreting them as constraints on the pair-production cross-section times branching fractions, we find that none of these searches constrain the $\Sigma \mathrm{SM} .{ }^{6}$ Note that this interpretation neglects the details of the analyses, i.e., ref. [78] specifically searches for, and places constraints on, $h^{ \pm} b \bar{b} W^{\mp}$ production, not general $h^{ \pm}$production. However, as the bound on the cross sections is too weak to constrain the $\Sigma \mathrm{SM}$, a more detailed examination is unnecessary.

There are dedicated pair production searches for neutral scalars with a focus on new contributions to SM Higgs pair production $[84,85]$. The signal regions in these analyses will constrain our model. However, as mentioned previously, the only source of neutral $h_{2} h_{2}$ pairs is via an off-shell $h_{1}$. While this process can give a $\sim 10 \%$ correction to the overall pair production cross section for large $\mu_{\Sigma}^{2}$, the cross section is too small to be constrained by these searches. Additionally, if the new charged scalars are heavy, $h_{1} h_{1}$ pairs could be produced via $h^{+} h^{-} \rightarrow h_{1} h_{1} W^{+} W^{-}$. However, once again the cross section and branching fractions are too small to be constrained by current SM Higgs pair production searches.

As pointed out by refs. $[8,86]$, there is a lack of dedicated searches for pair production involving charged scalars at $13 \mathrm{TeV}$. In particular, there are no recent searches with $t \bar{b}, \bar{t} b$ final states, which might arise in the triplet model via $h^{+} h^{-}$pair production if $m_{h^{ \pm}}>m_{t}$. Similarly, there are no recent searches with $t \bar{t}, t \bar{b}(\bar{t} b)$ or $b \bar{b}, t \bar{b}(\bar{t} b)$ final states, which may arise in $h_{2} h^{ \pm}$pair production. The latter of these final states is explored in ref. [8]. However, for $m_{h_{2}} \gtrsim 150 \mathrm{GeV}$ our branching fraction $\operatorname{Br}\left(h_{2} \rightarrow b \bar{b}\right)$ becomes too small for this final state to constrain the minimal triplet model.

There are other LHC searches that feature similar final states that can be used to constrain the $\Sigma$ SM. In particular, note that when the triplets are light $\left(m_{h_{2}} \lesssim 110 \mathrm{GeV}\right)$ $h_{2} h^{ \pm}$production can result in $\tau \tau \tau \nu_{\tau}$ pairs. On the other hand, for heavy triplets, processes such as $h^{+} h^{-} \rightarrow W^{+} W^{-} Z Z$ or $h_{2} h^{ \pm} \rightarrow W^{+} W^{-} W^{ \pm} Z$ can lead to a large number of leptons if some of the weak gauge bosons decay leptonically. Therefore searches featuring multilepton signal regions can be used to place constraints on the $\Sigma S M$.

\subsection{Collider constraints}

We utilise the CheckMATE 2.0.26 [87] package in order to examine the constraints arising from multilepton collider searches. CheckMATE compares simulated collider events against a range of CMS and ATLAS analyses and determines whether a given model is excluded.

\footnotetext{
${ }^{6}$ Except for a small region of parameter-space with a large diphoton branching fraction, which is discussed in more detail in the next section.
} 
We utilise MadGraph5 to generate parton level pair production events, with the production processes described in section 4.1. These events are showered by Pythia 8.230 [88] and are then run through the Delphes 3.4.1 [89] detector simulation using the CheckMATE interface. CheckMATE then evaluates the $\mathrm{CL}_{\mathrm{S}}[90]$ value for every signal region in each of the implemented CMS and ATLAS analyses and uses the most sensitive signal region to determine whether a model is excluded or not. These tools are dependent on a variety of other packages and tools [91-99].

The most constraining analyses are generally ATLAS or CMS searches for charginos and neutralinos with multilepton final states, specifically the searches in refs. [100] and [101], each using $36 \mathrm{fb}^{-1}$ of data taken at $13 \mathrm{TeV}$. Additionally, as mentioned in previous sections, the diphoton branching fraction for the new scalar can be large if $\mu_{\Sigma}^{2}=v_{\Sigma}^{2} b_{4}$ and $m_{h_{2}} \lesssim m_{W}$. Hence these parameter points are excluded by analyses with photonic signatures, such as ref. [102]. Note that this region of parameter space is also excluded by direct diphoton resonance searches, which are not yet implemented in checkmate [73, 74].

We varied the mass of the triplet-like neutral scalar $m_{h_{2}}$ from 70 to $350 \mathrm{GeV}$ in steps of $10 \mathrm{GeV}$. We let $\mu_{\Sigma}^{2}$ range from $-100^{2}$ to $200^{2} \mathrm{GeV}^{2}$. For $m_{h_{2}}<150 \mathrm{GeV}$ and $m_{h_{2}} \geq$ $150 \mathrm{GeV}$, we let $\sqrt{\left|\mu_{\Sigma}^{2}\right|}$ vary in steps of 25 and $100 \mathrm{GeV}$, respectively. The triplet quartic coupling and VEV were set to 1 and $1 \mathrm{GeV}$, respectively. Note that setting $b_{4}=1$ violates eq. (2.7) for large values of $\mu_{\Sigma}^{2}$. However, $b_{4}$ has negligible impact on collider phenomenology when $\mu_{\Sigma}^{2}$ is large, such that the results are independent of the choice of $b_{4}$. In order to increase the fraction of generated events resulting in signal events for parameter points with $m_{h_{2}} \leq 100 \mathrm{GeV}$ and $\left|\mu_{\Sigma}^{2}\right| \geq 50 \mathrm{GeV}^{2}$, the triplet-like scalars were forced to decay into $\tau$ leptons using MadSpin [103]. Outside of this region of parameter space all decays were allowed. Five million pair production events were generated for most parameter sets. Ten million events were generated for points near the $95 \%$ exclusion boundary.

The resulting $\mathrm{CL}_{\mathrm{S}}$-values obtained by CheckMATE are shown in figure 10. From the figure, we see that an unstable triplet-like scalar is required to have a mass $m_{h_{2}} \gtrsim 230 \mathrm{GeV}$. The one exception is a region of the parameter space near $m_{h_{2}}=120 \mathrm{GeV}$ and $\mu_{\Sigma}^{2}=$ $50^{2} \mathrm{GeV}^{2}$, which is only excluded at $84 \%$ confidence. As seen in figures 8 and 9, masses near $120 \mathrm{GeV}$ correspond to the transition between weak gauge boson and fermion pair decays, with the branching fraction of $h_{2} \rightarrow \tau^{+} \tau^{-}$and $h^{+} \rightarrow \tau^{+} \nu_{\tau}$ decreasing. Furthermore, the rate of $h_{2} \rightarrow W^{ \pm}\left(W^{\mp}\right)^{*}$ is proportional to $4 \frac{v_{\Sigma}}{v_{H}} \cos \theta_{N}+\sin \theta_{N}$, which goes to zero near $\mu_{\Sigma}^{2}=50^{2} \mathrm{GeV}^{2}$ and $m_{h_{2}}=120 \mathrm{GeV}$. Both of these factors combined lead to slightly fewer signal events near $m_{h_{2}}=120 \mathrm{GeV}$ and $\mu_{\Sigma}^{2}=50^{2} \mathrm{GeV}^{2}$. Thus this region of parameter space is not quite excluded by CheckMATE. Note that CheckMATE determines its $\mathrm{CL}_{\mathrm{S}}$ values using only the signal region that has the best sensitivity assuming the observed number of events match the SM prediction. This is done in order to avoid falsely excluding a model due to a downward fluctuation in the observed number of events. However, it should be noted that while the most sensitive signal region, region I04 in ref. [100], does not exclude this point, three other signal regions from the same analysis (C18, G03, G05) each individually exclude this point at $94 \%$ confidence. We have also utilised HiggsBounds 5.3.2beta [104- 


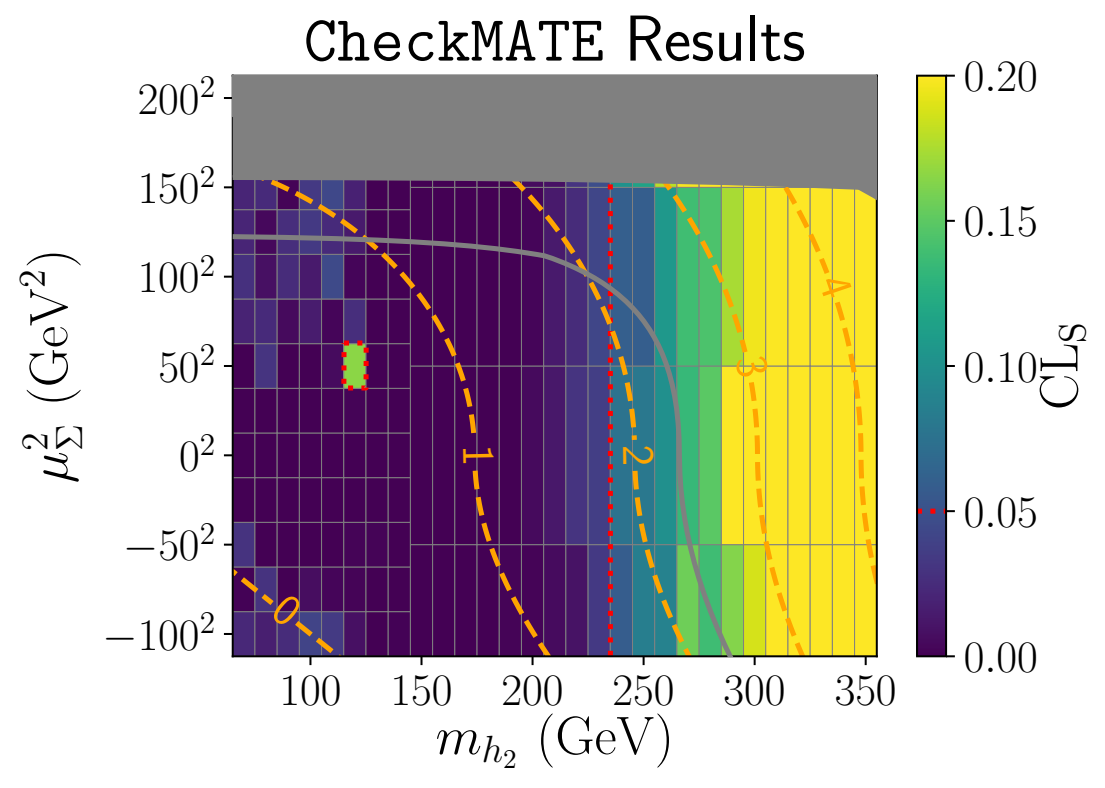

Figure 10. CheckMATE $\mathrm{CL}_{\mathrm{S}}$ exclusion values evaluated on a grid of masses and quadratic terms with $v_{\Sigma}=1 \mathrm{GeV}$ and $b_{4}=1$. Points with $\mathrm{CL}_{\mathrm{S}} \leq 0.05$ are excluded at $95 \%$ confidence. The $\mathrm{CL}_{\mathrm{S}}=0.05$ contour is indicated by a dashed red line. The dotted orange lines are contours of constant triplet-Higgs coupling. The solid grey region is the parameter-space where the scalar couplings become non-perturbative at energies $\Lambda<1 \mathrm{TeV}$, and the solid grey line shows where this contour would be if the cutoff energy is increased to $\Lambda=10^{6} \mathrm{GeV}$. The perturbativity contour does not use $b_{4}=1$, and instead selects $b_{4}$ as described for figure 1 .

108] and HiggsSignals 2.2.3beta [109-111] in order to verify that this parameter point is not separately excluded by dedicated new scalar searches or corrections to SM Higgs signals.

Recently new searches with multilepton signals have been released that utilise up to $139 \mathrm{fb}^{-1}$ of data [112-115]. These analyses have not yet been implemented in CheckMATE. Based on a simple scaling approximation using the Collider-Reach tool [116], we expect that this will increase the lower bound on the triplet mass to above $m_{h_{2}} \sim 330 \mathrm{GeV}$, and we expect the small allowed region to become excluded.

These collider constraints significantly restrict the parameter-space available for novel multi-step electroweak baryogenesis models. In particular, the parameter-space considered in ref. [3], and a significant chunk of the parameter-space considered in refs. [8, 17], are excluded by these constraints. We expect that other models featuring $\mathrm{SU}(2)$ triplet scalars decaying in such a manner would be similarly constrained.

Note that we have only considered values of the triplet VEV that result in short-lived triplets. In the limit where the triplet VEV approaches zero $\left(v_{\Sigma} \lesssim 10^{-4} \mathrm{GeV}[18]\right)$, the decays of the scalars will once again resemble those in the $\mathbb{Z}_{2}$ symmetric case; the $h_{2}$ will be stable on detector timescales and the $h^{ \pm}$will decay into $h_{2} \pi^{ \pm}$or $h_{2} \ell \nu$. At this point disappearing tracks will once again constrain the triplet, though dark matter direct detection constraints are avoided. For some small range of $v_{\Sigma}$ (or equivalently $a_{1}$ ) the decays of $h_{2}$ will be displaced from the primary vertex but still inside the detector. In this 
case both the disappearing charged track and multilepton searches will lose their efficacy. The detailed phenomenology of this intermediate regime is worth a study in its own right, and could be constrained through searches for displaced jets and leptons such as [117, 118]. Displaced vertex searches for scalars have been considered in the context of type-II seesaw models [31]. However, we are unaware of any such search for the minimal hypercharge-zero triplet scalar model.

\section{Conclusion}

Taking to heart the notion that electroweak baryogenesis is attractive for its testability at the LHC and prospective future colliders, we have examined the phenomenology of light $\mathrm{SU}(2)$ real triplet scalars motivated by multi-step electroweak phase transitions. We have demonstrated that such scalars are nearly excluded if they are stable. The only region of parameter-space still allowed is where the magnitude of the Higgs portal coupling $<1$, which is unsuitable for a two-step electroweak phase transition. This constraint can be avoided by breaking the $\mathbb{Z}_{2}$ symmetry that stabilises the neutral component of the triplet, allowing it to decay. However, depending on the lifetime of the charged triplets, one must then contend with either disappearing track or multilepton searches at colliders. These searches constrain the mass of the triplet to be at least 250 or $230 \mathrm{GeV}$, respectively. It may be possible that there is a region of parameter-space in-between the two extremes where both search types lose sensitivity. However, this likely requires a finely tuned selection for the triplet VEV.

It should be noted that electroweak baryogenesis in the presence of a real triplet scalar extension of the Standard Model requires particle content beyond the $\Sigma$ SM. In particular, the $\Sigma$ SM provides no additional sources of CP violation. Therefore, any realistic electroweak baryogenesis model will necessarily feature additional particles which may couple to the real scalar triplet, as is the case in ref. [6]. In addition to the phenomenology introduced by the new particle content, the decay channels of the triplets would likely also be modified such that the results obtained here will not be directly applicable. However, the collider constraints on the triplet parameter-space will likely be similarly restrictive. Alternatively, the constraints imposed on the scalar potential could be relaxed by considering further extensions of the scalar sector. This might allow for a large negative $\mu_{\Sigma}^{2}$ term, such that the triplet can be heavier at zero temperature.

\section{Acknowledgments}

We thank Giovanna Cottin and Yong Du for helpful discussion of the disappearing charged track searches. This work was supported in part by the Australian Research Council. Feynman diagrams were drawn using the TikZ-Feynman package [119]. The work of MJRM was supported in part under U.S. Department of Energy Contract DE-SC0011095 and National Natural Science Foundation of China grant number 19Z103010239. 
Open Access. This article is distributed under the terms of the Creative Commons Attribution License (CC-BY 4.0), which permits any use, distribution and reproduction in any medium, provided the original author(s) and source are credited.

\section{References}

[1] M.J. Ramsey-Musolf, The electroweak phase transition: a collider target, arXiv: 1912.07189 [INSPIRE].

[2] D.E. Morrissey and M.J. Ramsey-Musolf, Electroweak baryogenesis, New J. Phys. 14 (2012) 125003 [arXiv: 1206. 2942] [INSPIRE].

[3] H.H. Patel and M.J. Ramsey-Musolf, Stepping into electroweak symmetry breaking: phase transitions and Higgs phenomenology, Phys. Rev. D 88 (2013) 035013 [arXiv:1212.5652] [INSPIRE].

[4] H.H. Patel, M.J. Ramsey-Musolf and M.B. Wise, Color breaking in the early universe, Phys. Rev. D 88 (2013) 015003 [arXiv: 1303.1140] [INSPIRE].

[5] N. Blinov, J. Kozaczuk, D.E. Morrissey and C. Tamarit, Electroweak baryogenesis from exotic electroweak symmetry breaking, Phys. Rev. D 92 (2015) 035012 [arXiv:1504.05195] [INSPIRE].

[6] S. Inoue, G. Ovanesyan and M.J. Ramsey-Musolf, Two-step electroweak baryogenesis, Phys. Rev. D 93 (2016) 015013 [arXiv: 1508.05404] [InSPIRE].

[7] M.J. Ramsey-Musolf, P. Winslow and G. White, Color breaking baryogenesis, Phys. Rev. D 97 (2018) 123509 [arXiv:1708.07511] [INSPIRE].

[8] M. Chala, M. Ramos and M. Spannowsky, Gravitational wave and collider probes of a triplet Higgs sector with a low cutoff, Eur. Phys. J. C 79 (2019) 156 [arXiv:1812.01901] [INSPIRE].

[9] S. Profumo, M.J. Ramsey-Musolf and G. Shaughnessy, Singlet Higgs phenomenology and the electroweak phase transition, JHEP 08 (2007) 010 [arXiv: 0705.2425] [INSPIRE].

[10] J.R. Espinosa, T. Konstandin and F. Riva, Strong electroweak phase transitions in the Standard Model with a singlet, Nucl. Phys. B 854 (2012) 592 [arXiv:1107.5441] [INSPIRE].

[11] D. Curtin, P. Meade and C.-T. Yu, Testing electroweak baryogenesis with future colliders, JHEP 11 (2014) 127 [arXiv:1409.0005] [INSPIRE].

[12] M. Jiang, L. Bian, W. Huang and J. Shu, Impact of a complex singlet: electroweak baryogenesis and dark matter, Phys. Rev. D 93 (2016) 065032 [arXiv:1502.07574] [INSPIRE].

[13] G. Kurup and M. Perelstein, Dynamics of electroweak phase transition in singlet-scalar extension of the Standard Model, Phys. Rev. D 96 (2017) 015036 [arXiv:1704.03381] [INSPIRE].

[14] C.-W. Chiang, M.J. Ramsey-Musolf and E. Senaha, Standard Model with a complex scalar singlet: cosmological implications and theoretical considerations, Phys. Rev. D 97 (2018) 015005 [arXiv: 1707.09960] [INSPIRE].

[15] N.F. Bell, M.J. Dolan, L.S. Friedrich, M.J. Ramsey-Musolf and R.R. Volkas, Electroweak baryogenesis with vector-like leptons and scalar singlets, JHEP 09 (2019) 012 [arXiv: 1903.11255] [INSPIRE]. 
[16] S.A.R. Ellis, T. Gherghetta, K. Kaneta and K.A. Olive, New weak-scale physics from $\mathrm{SO}(10)$ with high-scale supersymmetry, Phys. Rev. D 98 (2018) 055009 [arXiv: 1807.06488] [INSPIRE].

[17] L. Niemi, H.H. Patel, M.J. Ramsey-Musolf, T.V.I. Tenkanen and D.J. Weir, Electroweak phase transition in the real triplet extension of the SM: dimensional reduction, Phys. Rev. D 100 (2019) 035002 [arXiv:1802.10500] [INSPIRE].

[18] P. Fileviez Perez, H.H. Patel, M. Ramsey-Musolf and K. Wang, Triplet scalars and dark matter at the LHC, Phys. Rev. D 79 (2009) 055024 [arXiv:0811.3957] [INSPIRE].

[19] L. Wang and X.-F. Han, LHC diphoton and $Z+$ photon Higgs signals in the Higgs triplet model with $Y=0$, JHEP 03 (2014) 010 [arXiv:1303.4490] [INSPIRE].

[20] N. Khan, Exploring the hyperchargeless Higgs triplet model up to the Planck scale, Eur. Phys. J. C 78 (2018) 341 [arXiv:1610.03178] [INSPIRE].

[21] M. Chabab, M.C. Peyranère and L. Rahili, Probing the Higgs sector of $Y=0$ Higgs triplet model at LHC, Eur. Phys. J. C 78 (2018) 873 [arXiv:1805.00286] [InSPIRE].

[22] M. Cirelli, N. Fornengo and A. Strumia, Minimal dark matter, Nucl. Phys. B 753 (2006) 178 [hep-ph/0512090] [INSPIRE].

[23] M. Cirelli, A. Strumia and M. Tamburini, Cosmology and astrophysics of minimal dark matter, Nucl. Phys. B 787 (2007) 152 [arXiv:0706.4071] [InSPIRE].

[24] M. Cirelli, R. Franceschini and A. Strumia, Minimal dark matter predictions for galactic positrons, anti-protons, photons, Nucl. Phys. B 800 (2008) 204 [arXiv:0802.3378] [INSPIRE].

[25] S.S. AbdusSalam and T.A. Chowdhury, Scalar representations in the light of electroweak phase transition and cold dark matter phenomenology, JCAP 05 (2014) 026 [arXiv:1310.8152] [INSPIRE].

[26] O. Fischer and J.J. van der Bij, The scalar singlet-triplet dark matter model, JCAP 01 (2014) 032 [arXiv:1311.1077] [INSPIRE].

[27] S. Yaser Ayazi and S.M. Firouzabadi, Constraining inert triplet dark matter by the LHC and FermiLAT, JCAP 11 (2014) 005 [arXiv:1408.0654] [INSPIRE].

[28] S. Yaser Ayazi and S.M. Firouzabadi, Footprint of triplet scalar dark matter in direct, indirect search and invisible Higgs decay, Cogent Phys. 2 (2015) 1047559 [arXiv: 1501.06176] [INSPIRE].

[29] P. Bandyopadhyay, K. Huitu and A. Sabanci Keceli, Multi-lepton signatures of the triplet like charged Higgs at the LHC, JHEP 05 (2015) 026 [arXiv: 1412.7359] [INSPIRE].

[30] M. Mitra, S. Niyogi and M. Spannowsky, Type-II seesaw model and multilepton signatures at hadron colliders, Phys. Rev. D 95 (2017) 035042 [arXiv:1611.09594] [INSPIRE].

[31] P.S. Bhupal Dev and Y. Zhang, Displaced vertex signatures of doubly charged scalars in the type-II seesaw and its left-right extensions, JHEP 10 (2018) 199 [arXiv:1808.00943] [INSPIRE].

[32] R. Primulando, J. Julio and P. Uttayarat, Scalar phenomenology in type-II seesaw model, JHEP 08 (2019) 024 [arXiv: 1903.02493] [INSPIRE].

[33] Particle Data Group collaboration, Review of particle physics, Phys. Rev. D 98 (2018) 030001 [INSPIRE]. 
[34] D.J. Griffiths, Introduction to elementary particles, $2^{\text {nd }}$ rev. version, Wiley, New York, NY, U.S.A. (2008).

[35] K. Riesselmann and S. Willenbrock, Ruling out a strongly interacting standard Higgs model, Phys. Rev. D 55 (1997) 311 [hep-ph/9608280] [InSPIRE].

[36] T. Hambye and K. Riesselmann, Matching conditions and Higgs mass upper bounds revisited, Phys. Rev. D 55 (1997) 7255 [hep-ph/9610272] [INSPIRE].

[37] M. Gonderinger, H. Lim and M.J. Ramsey-Musolf, Complex scalar singlet dark matter: vacuum stability and phenomenology, Phys. Rev. D 86 (2012) 043511 [arXiv:1202.1316] [INSPIRE].

[38] Y. Du, A. Dunbrack, M.J. Ramsey-Musolf and J.-H. Yu, Type-II seesaw scalar triplet model at a 100 TeV pp collider: discovery and Higgs portal coupling determination, JHEP 01 (2019) 101 [arXiv: 1810.09450] [INSPIRE].

[39] F. Staub, SARAH 4: a tool for (not only SUSY) model builders, Comput. Phys. Commun. 185 (2014) 1773 [arXiv: 1309.7223] [INSPIRE].

[40] H.H. Patel and M.J. Ramsey-Musolf, Baryon washout, electroweak phase transition and perturbation theory, JHEP 07 (2011) 029 [arXiv:1101.4665] [INSPIRE].

[41] O. Gould, J. Kozaczuk, L. Niemi, M.J. Ramsey-Musolf, T.V.I. Tenkanen and D.J. Weir, Nonperturbative analysis of the gravitational waves from a first-order electroweak phase transition, Phys. Rev. D 100 (2019) 115024 [arXiv:1903.11604] [INSPIRE].

[42] A. Djouadi, The anatomy of electro-weak symmetry breaking. I: the Higgs boson in the Standard Model, Phys. Rept. 457 (2008) 1 [hep-ph/0503172] [INSPIRE].

[43] ATLAS collaboration, Measurements of Higgs boson properties in the diphoton decay channel with $36 \mathrm{fb}^{-1}$ of pp collision data at $\sqrt{\mathrm{s}}=13 \mathrm{TeV}$ with the ATLAS detector, Phys. Rev. D 98 (2018) 052005 [arXiv: 1802.04146] [INSPIRE].

[44] CMS collaboration, Measurements of Higgs boson properties in the diphoton decay channel in proton-proton collisions at $\sqrt{s}=13 \mathrm{TeV}$, JHEP 11 (2018) 185 [arXiv:1804.02716] [INSPIRE].

[45] ATLAS collaboration, Projections for measurements of Higgs boson cross sections, branching ratios and coupling parameters with the ATLAS detector at a HL-LHC, ATL-PHYS-PUB-2013-014, CERN, Geneva, Switzerland (2013).

[46] CMS collaboration, Projected performance of Higgs analyses at the HL-LHC for ECFA 2016, CMS-PAS-FTR-16-002, CERN, Geneva, Switzerland (2017).

[47] CMS collaboration, Search for disappearing tracks as a signature of new long-lived particles in proton-proton collisions at $\sqrt{s}=13 \mathrm{TeV}, \mathrm{JHEP} 08$ (2018) 016 [arXiv:1804.07321] [INSPIRE].

[48] ATLAS collaboration, Search for long-lived charginos based on a disappearing-track signature in pp collisions at $\sqrt{s}=13 \mathrm{TeV}$ with the ATLAS detector, JHEP 06 (2018) 022 [arXiv: 1712.02118] [INSPIRE].

[49] ATLAS collaboration, Upper limit EW of search for long-lived charginos based on a disappearing-track signature in pp collisions at $\sqrt{s}=13 \mathrm{TeV}$ with the ATLAS detector, CERN, Geneva, Switzerland (2018). 
[50] J. Alwall et al., The automated computation of tree-level and next-to-leading order differential cross sections and their matching to parton shower simulations, JHEP 07 (2014) 079 [arXiv: 1405.0301] [inSPIRE].

[51] C. Degrande, C. Duhr, B. Fuks, D. Grellscheid, O. Mattelaer and T. Reiter, UFO - the Universal FeynRules Output, Comput. Phys. Commun. 183 (2012) 1201 [arXiv:1108.2040] [INSPIRE].

[52] A. Alloul, N.D. Christensen, C. Degrande, C. Duhr and B. Fuks, FeynRules $2.0-a$ complete toolbox for tree-level phenomenology, Comput. Phys. Commun. 185 (2014) 2250 [arXiv:1310.1921] [INSPIRE].

[53] T. Hahn, Generating Feynman diagrams and amplitudes with FeynArts 3, Comput. Phys. Commun. 140 (2001) 418 [hep-ph/0012260] [INSPIRE].

[54] N.D. Christensen et al., A comprehensive approach to new physics simulations, Eur. Phys. J. C 71 (2011) 1541 [arXiv:0906.2474] [InSPIRE].

[55] C. Degrande, Automatic evaluation of $U V$ and $R_{2}$ terms for beyond the Standard Model Lagrangians: a proof-of-principle, Comput. Phys. Commun. 197 (2015) 239 [arXiv:1406.3030] [INSPIRE].

[56] A. Pierce and J. Thaler, Natural dark matter from an unnatural Higgs boson and new colored particles at the TeV scale, JHEP 08 (2007) 026 [hep-ph/0703056] [INSPIRE].

[57] D. Egana-Ugrinovic, M. Low and J.T. Ruderman, Charged fermions below $100 \mathrm{GeV}$, JHEP 05 (2018) 012 [arXiv: 1801.05432] [INSPIRE].

[58] J. McKay, P. Scott and P. Athron, Pitfalls of iterative pole mass calculation in electroweak multiplets, Eur. Phys. J. Plus 133 (2018) 444 [arXiv:1710.01511] [InSPIRE].

[59] J. McKay and P. Scott, Two-loop mass splittings in electroweak multiplets: winos and minimal dark matter, Phys. Rev. D 97 (2018) 055049 [arXiv:1712.00968] [INSPIRE].

[60] G. Bélanger, F. Boudjema, A. Goudelis, A. Pukhov and B. Zaldivar, MicrOMEGAs5.0: freeze-in, Comput. Phys. Commun. 231 (2018) 173 [arXiv:1801.03509] [InSPIRE].

[61] Planck collaboration, Planck 2018 results. VI. Cosmological parameters, arXiv: 1807.06209 [INSPIRE].

[62] F. Ambrogi et al., MadDM v.3.0: a comprehensive tool for dark matter studies, Phys. Dark Univ. 24 (2019) 100249 [arXiv: 1804.00044] [INSPIRE].

[63] M. D'Onofrio, K. Rummukainen and A. Tranberg, Sphaleron rate in the minimal Standard Model, Phys. Rev. Lett. 113 (2014) 141602 [arXiv: 1404.3565] [INSPIRE].

[64] W. Chao, G.-J. Ding, X.-G. He and M. Ramsey-Musolf, Scalar electroweak multiplet dark matter, JHEP 08 (2019) 058 [arXiv: 1812.07829] [INSPIRE].

[65] XENON collaboration, Dark matter search results from a one ton-year exposure of XENON1T, Phys. Rev. Lett. 121 (2018) 111302 [arXiv:1805.12562] [INSPIRE].

[66] M.J. Dolan, C. Englert and M. Spannowsky, Higgs self-coupling measurements at the LHC, JHEP 10 (2012) 112 [arXiv:1206.5001] [InSPIRE].

[67] C. Anastasiou, C. Duhr, F. Dulat, F. Herzog and B. Mistlberger, Higgs boson gluon-fusion production in QCD at three loops, Phys. Rev. Lett. 114 (2015) 212001 [arXiv:1503.06056] [INSPIRE]. 
[68] S. Dawson, S. Dittmaier and M. Spira, Neutral Higgs boson pair production at hadron colliders: QCD corrections, Phys. Rev. D 58 (1998) 115012 [hep-ph/9805244] [INSPIRE].

[69] D. de Florian et al., Differential Higgs boson pair production at next-to-next-to-leading order in QCD, JHEP 09 (2016) 151 [arXiv:1606.09519] [INSPIRE].

[70] M. Grazzini et al., Higgs boson pair production at NNLO with top quark mass effects, JHEP 05 (2018) 059 [arXiv: 1803.02463] [INSPIRE].

[71] A. Djouadi, J. Kalinowski, M. Muehlleitner and M. Spira, HDECAY: twenty++ years after, Comput. Phys. Commun. 238 (2019) 214 [arXiv:1801.09506] [INSPIRE].

[72] J. Alwall, C. Duhr, B. Fuks, O. Mattelaer, D.G. Öztürk and C.-H. Shen, Computing decay rates for new physics theories with FeynRules and MadGraph5_aMC@NLO, Comput. Phys. Commun. 197 (2015) 312 [arXiv:1402.1178] [INSPIRE].

[73] ATLAS collaboration, Search for resonances in the 65 to $110 \mathrm{GeV}$ diphoton invariant mass range using $80 \mathrm{fb}^{-1}$ of pp collisions collected at $\sqrt{\mathrm{s}}=13 \mathrm{TeV}$ with the ATLAS detector, ATLAS-CONF-2018-025, CERN, Geneva, Switzerland (2018).

[74] CMS collaboration, Search for a Standard Model-like Higgs boson in the mass range between 70 and $110 \mathrm{GeV}$ in the diphoton final state in proton-proton collisions at $\sqrt{s}=8$ and 13 TeV, Phys. Lett. B 793 (2019) 320 [arXiv:1811.08459] [InSPIRE].

[75] ATLAS collaboration, Search for new phenomena in high-mass diphoton final states using $37 \mathrm{fb}^{-1}$ of proton-proton collisions collected at $\sqrt{\mathrm{s}}=13 \mathrm{TeV}$ with the ATLAS detector, Phys. Lett. B 775 (2017) 105 [arXiv: 1707.04147] [INSPIRE].

[76] CMS collaboration, Search for physics beyond the Standard Model in high-mass diphoton events from proton-proton collisions at $\sqrt{s}=13$ TeV, Phys. Rev. D 98 (2018) 092001 [arXiv: 1809.00327] [INSPIRE].

[77] CMS collaboration, Search for additional neutral MSSM Higgs bosons in the $\tau \tau$ final state in proton-proton collisions at $\sqrt{s}=13 \mathrm{TeV}$, JHEP 09 (2018) 007 [arXiv:1803.06553] [INSPIRE].

[78] ATLAS collaboration, Search for charged Higgs bosons decaying via $H^{ \pm} \rightarrow \tau^{ \pm} \nu_{\tau}$ in the $\tau+$ jets and $\tau+$ lepton final states with $36 \mathrm{fb}^{-1}$ of pp collision data recorded at $\sqrt{\mathrm{s}}=13 \mathrm{TeV}$ with the ATLAS experiment, JHEP 09 (2018) 139 [arXiv: 1807.07915] [INSPIRE].

[79] CMS collaboration, Search for charged Higgs bosons in the $H^{ \pm} \rightarrow \tau^{ \pm} \nu_{\tau}$ decay channel in proton-proton collisions at $\sqrt{s}=13 \mathrm{TeV}$, JHEP 07 (2019) 142 [arXiv:1903.04560] [INSPIRE].

[80] CMS collaboration, Search for a charged Higgs boson decaying into top and bottom quarks in events with electrons or muons in proton-proton collisions at $\sqrt{s}=13 \mathrm{TeV}$, JHEP 01 (2020) 096 [arXiv: 1908.09206] [inSPIRE].

[81] ATLAS collaboration, Search for charged Higgs bosons decaying into top and bottom quarks at $\sqrt{s}=13 \mathrm{TeV}$ with the ATLAS detector, JHEP 11 (2018) 085 [arXiv:1808.03599] [INSPIRE].

[82] ATLAS collaboration, Search for a charged Higgs boson produced in the vector-boson fusion mode with decay $H^{ \pm} \rightarrow W^{ \pm} Z$ using pp collisions at $\sqrt{s}=8 \mathrm{TeV}$ with the ATLAS experiment, Phys. Rev. Lett. 114 (2015) 231801 [arXiv:1503.04233] [INSPIRE]. 
[83] CMS collaboration, Search for charged Higgs bosons produced via vector boson fusion and decaying into a pair of $W$ and $Z$ bosons using pp collisions at $\sqrt{s}=13$ TeV, Phys. Rev. Lett. 119 (2017) 141802 [arXiv:1705.02942] [INSPIRE].

[84] CMS collaboration, Combination of searches for Higgs boson pair production in proton-proton collisions at $\sqrt{s}=13 \mathrm{TeV}$, Phys. Rev. Lett. 122 (2019) 121803 [arXiv: 1811.09689] [INSPIRE].

[85] ATLAS collaboration, Combination of searches for Higgs boson pairs in pp collisions at $\sqrt{s}=13 \mathrm{TeV}$ with the ATLAS detector, Phys. Lett. B 800 (2020) 135103 [arXiv: 1906. 02025] [INSPIRE].

[86] G.D. Kribs, A. Martin, B. Ostdiek and T. Tong, Dark mesons at the LHC, JHEP 07 (2019) 133 [arXiv: 1809.10184] [INSPIRE].

[87] D. Dercks, N. Desai, J.S. Kim, K. Rolbiecki, J. Tattersall and T. Weber, CheckMATE 2: from the model to the limit, Comput. Phys. Commun. 221 (2017) 383 [arXiv:1611.09856] [INSPIRE].

[88] T. Sjöstrand et al., An introduction to PYTHIA 8.2, Comput. Phys. Commun. 191 (2015) 159 [arXiv: 1410.3012] [INSPIRE].

[89] DELPHES 3 collaboration, DELPHES 3, a modular framework for fast simulation of a generic collider experiment, JHEP 02 (2014) 057 [arXiv: 1307.6346] [INSPIRE].

[90] A.L. Read, Presentation of search results: the $C L_{s}$ technique, J. Phys. G 28 (2002) 2693 [INSPIRE].

[91] M. Dobbs and J.B. Hansen, The HepMC C++ Monte Carlo event record for high energy physics, Comput. Phys. Commun. 134 (2001) 41 [INSPIRE].

[92] M. Cacciari and G.P. Salam, Dispelling the $N^{3}$ myth for the $k_{t}$ jet-finder, Phys. Lett. B 641 (2006) 57 [hep-ph/0512210] [INSPIRE].

[93] M. Cacciari, G.P. Salam and G. Soyez, The anti-k $k_{t}$ jet clustering algorithm, JHEP 04 (2008) 063 [arXiv:0802.1189] [INSPIRE].

[94] R. Frederix, S. Frixione, F. Maltoni and T. Stelzer, Automation of next-to-leading order computations in QCD: the FKS subtraction, JHEP 10 (2009) 003 [arXiv:0908.4272] [INSPIRE].

[95] V. Hirschi, R. Frederix, S. Frixione, M.V. Garzelli, F. Maltoni and R. Pittau, Automation of one-loop QCD corrections, JHEP 05 (2011) 044 [arXiv:1103.0621] [INSPIRE].

[96] M. Cacciari, G.P. Salam and G. Soyez, FastJet user manual, Eur. Phys. J. C 72 (2012) 1896 [arXiv:1111.6097] [INSPIRE].

[97] R. Frederix, S. Frixione, V. Hirschi, F. Maltoni, R. Pittau and P. Torrielli, Four-lepton production at hadron colliders: aMC@NLO predictions with theoretical uncertainties, JHEP 02 (2012) 099 [arXiv: 1110.4738] [INSPIRE].

[98] A. Buckley et al., LHAPDF6: parton density access in the LHC precision era, Eur. Phys. J. C 75 (2015) 132 [arXiv:1412.7420] [INSPIRE].

[99] V. Hirschi and O. Mattelaer, Automated event generation for loop-induced processes, JHEP 10 (2015) 146 [arXiv:1507.00020] [INSPIRE]. 
[100] CMS collaboration, Search for electroweak production of charginos and neutralinos in multilepton final states in proton-proton collisions at $\sqrt{s}=13$ TeV, JHEP 03 (2018) 166 [arXiv:1709.05406] [INSPIRE].

[101] ATLAS collaboration, Search for the direct production of charginos and neutralinos in final states with tau leptons in $\sqrt{s}=13$ TeV pp collisions with the ATLAS detector, Eur. Phys.

J. C 78 (2018) 154 [arXiv:1708.07875] [InSPIRE].

[102] ATLAS collaboration, Search for photonic signatures of gauge-mediated supersymmetry in 13 TeV pp collisions with the ATLAS detector, Phys. Rev. D 97 (2018) 092006 [arXiv: 1802.03158] [INSPIRE].

[103] P. Artoisenet, R. Frederix, O. Mattelaer and R. Rietkerk, Automatic spin-entangled decays of heavy resonances in Monte Carlo simulations, JHEP 03 (2013) 015 [arXiv:1212.3460] [INSPIRE].

[104] P. Bechtle, O. Brein, S. Heinemeyer, G. Weiglein and K.E. Williams, HiggsBounds: confronting arbitrary Higgs sectors with exclusion bounds from LEP and the Tevatron, Comput. Phys. Commun. 181 (2010) 138 [arXiv:0811.4169] [InSPIRE].

[105] P. Bechtle, O. Brein, S. Heinemeyer, G. Weiglein and K.E. Williams, HiggsBounds 2.0.0: confronting neutral and charged Higgs sector predictions with exclusion bounds from LEP and the Tevatron, Comput. Phys. Commun. 182 (2011) 2605 [arXiv:1102.1898] [InSPIRE].

[106] P. Bechtle et al., Recent developments in HiggsBounds and a preview of HiggsSignals, PoS (CHARGED2012) 024 (2012) [arXiv:1301.2345] [INSPIRE].

[107] P. Bechtle et al., HiggsBounds-4: improved tests of extended Higgs sectors against exclusion bounds from LEP, the Tevatron and the LHC, Eur. Phys. J. C 74 (2014) 2693 [arXiv:1311.0055] [INSPIRE].

[108] P. Bechtle, S. Heinemeyer, O. Stal, T. Stefaniak and G. Weiglein, Applying exclusion likelihoods from LHC searches to extended Higgs sectors, Eur. Phys. J. C 75 (2015) 421 [arXiv: 1507.06706] [INSPIRE].

[109] P. Bechtle, S. Heinemeyer, O. Stål, T. Stefaniak and G. Weiglein, HiggsSignals: confronting arbitrary Higgs sectors with measurements at the Tevatron and the LHC, Eur. Phys. J. C 74 (2014) 2711 [arXiv: 1305.1933] [INSPIRE].

[110] O. Stål and T. Stefaniak, Constraining extended Higgs sectors with HiggsSignals, PoS (EPS-HEP2013) 314 (2013) [arXiv:1310.4039] [INSPIRE].

[111] P. Bechtle, S. Heinemeyer, O. Stål, T. Stefaniak and G. Weiglein, Probing the Standard Model with Higgs signal rates from the Tevatron, the $L H C$ and a future $I L C, J H E P 11$ (2014) 039 [arXiv: 1403.1582] [INSPIRE].

[112] CMS collaboration, Search for physics beyond the Standard Model in events with two same-sign leptons or at least three leptons and jets in proton-proton collisions at $\sqrt{s}=13$ TeV, CMS-PAS-SUS-19-008, CERN, Geneva, Switzerland (2019).

[113] CMS collaboration, Search for vector-like leptons in multilepton final states in proton-proton collisions at $\sqrt{s}=13$ TeV, Phys. Rev. D 100 (2019) 052003 [arXiv: 1905.10853] [INSPIRE].

[114] ATLAS collaboration, Search for chargino-neutralino production with mass splittings near the electroweak scale in three-lepton final states in $\sqrt{s}=13$ TeV pp collisions with the ATLAS detector, ATLAS-CONF-2019-020, CERN, Geneva, Switzerland (2019). 
[115] ATLAS collaboration, Search for squarks and gluinos in final states with same-sign leptons and jets using $139 \mathrm{fb}^{-1}$ of data collected with the ATLAS detector, arXiv:1909.08457 [INSPIRE].

[116] G. Salam and A. Weiler, Collider reach, http://collider-reach.web.cern.ch/collider-reach/.

[117] CMS collaboration, Search for long-lived particles decaying into displaced jets in proton-proton collisions at $\sqrt{s}=13$ TeV, Phys. Rev. D 99 (2019) 032011 [arXiv: 1811.07991] [INSPIRE].

[118] ATLAS collaboration, Search for displaced vertices of oppositely charged leptons from decays of long-lived particles in pp collisions at $\sqrt{s}=13 \mathrm{TeV}$ with the ATLAS detector, Phys. Lett. B 801 (2020) 135114 [arXiv:1907.10037] [INSPIRE].

[119] J. Ellis, TikZ-Feynman: Feynman diagrams with TikZ, Comput. Phys. Commun. 210 (2017) 103 [arXiv: 1601.05437] [INSPIRE]. 